\title{
Quality Evaluation of the Clinical Trials for Natural Products Used in Cancer: An Evidence-Based Literature Review
}

\author{
Rizwan Ahmad ${ }^{1, *}++^{(D)}$, Lina Hussain AlLehaibi ${ }^{2,+}$, Abdulrahman K. Alshammari ${ }^{3}$ and \\ Saif M. Alkhaldi 4 \\ 1 Natural Products and Alternative Medicines, College of Clinical Pharmacy, Imam Abdul, \\ Rahman Bin Faisal University, P.O. Box. 1982, Dammam 31441, Saudi Arabia \\ 2 First Health Cluster in Eastern Province, Dammam Medical Complex, Dammam 32245, Saudi Arabia; \\ Leena.alluhaibi@gmail.com or Lallehaibi@moh.gov.sa \\ 3 Rafha Central Hospital, Northern Border Region, Rafha City 91911, Saudi Arabia; Ab.khllaf@gmail.com \\ 4 King Khalid Hospital, Riyadh Region, Majma'ah City 11952, Saudi Arabia; ph.saif.alkhaldi@gmail.com \\ * Correspondence: rareiyadh@iau.edu.sa or rizvistar_36@yahoo.com; Tel.: +966-54-983-1835 \\ + Contributed equally in this review.
}

Received: 13 October 2020; Accepted: 5 November 2020; Published: 10 November 2020

\begin{abstract}
The amount of data regarding the use of herbs/herbal products in cancer clinical trials at times creates a great challenge for oncologists to prescribe or counsel patients. It urges critical evaluation of the quality of clinical trials. Herein, for the first time, the clinical trials for herbs used in cancer were critically evaluated on the basis of three widely used scales, i.e., Jadad, Delphi, and Cochrane scales. The literature was collected with the help of online databases, journals, libraries, and books using a number of specific keywords as mentioned in detail in forthcoming sections. A total of 73 clinical trials were extracted, evaluated, and scored for 14 herbs, according to the predefined criteria mentioned below. A major deficiency of "non-blinding of clinical trials" was observed. The principal component analysis revealed four components (PC1-PC4) with a total variability of $68.21 \%$, wherein the highest percentage variability was observed for PC1 loaded with "non-blinding of the clinical trials, no concealment of the treatment allocation, non-blindness of the patient and care provider", which accounted for $30.81 \%$ of the total variability. The next major variability of $14.70 \%$ was observed for PC2 loaded with "non-randomization of the studies, non-blinding of the outcome assessors, no proper drop-out procedures, and lack of information regarding baseline characteristics for the groups". Pearson's correlation further confirmed a similar correlation pattern for the mentioned deficiencies $(p=0.05)$. An in-house grading scale was developed, showing a very small portion (16.44\%), i.e., 12/73 studies with a good quality, whereas the majority (57.54\%) of the studies, i.e., $42 / 73$, were found to be of poor quality. The rules and regulations governing the quality of clinical trials needs to be more stringent and updated for the natural products/herbs used in cancer clinical trials.
\end{abstract}

Keywords: cancer; Jadad; Delphi; Cochrane; quality evaluation

\section{Introduction}

Cancer is a term implicated for an uncontrolled cell division that may invade nearby tissues and spread to other body parts via blood and lymph systems [1]. The major risk factors for this disease include age, family history, hormones, tobacco use, irradiations, chronic inflammation, diet, and sedentary lifestyle [2,3]. A study reported 1,735,350 cancer cases in the United Stated for the year 2018 , with total deaths of 609,640 [4]. The new estimates for 2040 revealed a global burden of up to 
27.5 million new cases with 16.3 million deaths [5]. Breast cancer is reported more prevalent among the type of cancers, however, cervix (Southern Asia, Africa), prostate (North America, Western Europe), and oral cancers (India) are also widely observed [6]. A range of treatment strategies such as surgery, systemic therapy, and radiation therapy are available for cancer treatment, but the disadvantages associated with these treatment exceeds their desired therapeutic outcomes. For instance, an increased wound complication with damage to surrounding tissues during radiation therapy, site-specific complications with more risks of infections after surgery, and systemic toxicities following systemic therapy are few of the uncontrolled circumstances reported after treatment $[7,8]$. On the contrary, the use herbs has shown a decreased incidence for cancer. Solanum nigrum and Cassia auriculata flowers [9], Aloe vera (radiation-induced mucositis) [10], Matricaria aurea (skin, prostate, breast, and ovarian cancer), Zingiber officinal, and Punica granatum are a few examples with promising growth inhibitory effects. [11-13] Even a number of potent active chemicals have been isolated from various genera such as Catharanthus, Taxus, Camptotheca, Curcuma, Betula, Podophyllum, and Cephalotaxus [14] and applied for anticancer activity, including the chemicals irinotecan (colorectal cancer), colchicine (leukemia and solid tumors), and cucurbitacin (various cancers) [15]. Clinical trials for herbs and herbal products are also increasing where studies are performed to evaluate the effectiveness and therapeutic safety. For instance, a study by Getz (1995-2005) reported an annual increase of new procedures (6.5\%), inclusion criteria (nearly thrice), and investigator site burden (10.5\%) in clinical trials. Ultimately, this huge burden on behalf of the investigator will adversely affect the performance [16]. An additional factor, underlined with a significant effect upon the clinical trial quality and compliance, is "globalization of clinical trials". An annual increase of $15 \%$ for the number of active investigators along with a twofold increase in the number of countries have been reported from 1995 to 2005 [17]. Interestingly, a major portion of these clinical trials is conducted in developing countries that have huge disparities in comparison with developed countries in terms of socioeconomic standards, education, and healthcare systems as well as differences in health infrastructure, medical training, and clinical practices and ethical values, which exerts a huge impact upon the quality of clinical trials. During the last few decades, a confronting challenge of increased cost along with a delay in new drug development has been faced in the global market, especially the pharmaceutical industry. To properly address the issue, with the best of solutions available for saving cost and time, the trend of clinical trial has been opted as one of the most promising tools. Although defining the quality of a clinical trial may be challenging at times, and the fact that no clinical trial can be perfect, it is nevertheless important to account for customer satisfaction with regards to needs and their expectations. Most of the time the outcomes of a clinical study may reach the general public, who may use these results as evidence for treating various diseases. Hence, it is important to assess the quality of clinical trials so to report a valid and authentic "quality clinical trial". Several systems are available to assess and evaluate the quality of a clinical trial. For instance, the Jadad score, final Delphi score, and Cochrane back review group score. The current study critically evaluates the clinical trials reported for natural products in cancer on an individual basis by using the three scales, wherein a score for each scale along with a final score in the three scales is calculated and reported in forthcoming sections. The scales are applied to each trial and the deficiencies observed per each scale are reported in a table below.

\section{Materials and Methods}

Databases and relevant literature search strategy: Journals: Cancer Research, Clinical Cancer Research, Journal of Clinical Oncology, Natural Product Research, Phytotherapy Research, Journal of Ethnopharmacology, BMC Complementary and Alternative Medicines. Databases: Science Direct, PubMed, SciFinder, Scopus, Google Scholar, e-resources, e-portal of Imam Abdulrahman Bin Faisal University library. Books: indigenous drugs of India, phytochemistry and ethnopharmacology, herbalism, etc.

The literature was thoroughly checked for duplication, as well as incomplete and ineligible study, as per inclusion criteria. The data were finalized, evaluated, and an individual as well as cumulative score was assigned to each clinical trial as per the points mentioned in the Jadad, Delphi, and Cochrane 
scale. Furthermore, an aggregate score was calculated and the quality of clinical trials was agreed on the basis of an in-house developed rating scale.

Keywords searched: The keywords searched for in literature included: randomized clinical trial, clinical trials, cancer, tumor, malignancy, aloe, Aloe vera, black seed, Nigella sativa, Boswellia, Boswellia serrata, chamomile, Matricaria suaveolens koch, colocynth, Citrullus lanatus, garlic, Allium sativum, ginger, Zingiber officinale, onion, Allium cepa, pomegranate, Punica granatum, senna, Cassia senna, Pistacia terebinthus, thyme, Thymus vulgaris, wheat, Triticum aestivum, Artemisia abaensis, Artemisia abbreviata, wormwood.

\subsection{Inclusion Criteria}

Inclusion criteria included clinical trials published in the English language; clinical trials using natural products and studies/reported in human subjects; any clinical trials using natural products with established folklore uses in cancer or with a reported use in a community worldwide (ethnopharmacological relevance); any clinical trial (phase I-V) reported for natural products in cancer, irrespective of blinding, randomization, statistical models, outcomes, and results presented; and all clinical trials using natural products along with conventional medication.

For ethnopharmacological relevance, a list of herbs/natural products was sorted and evaluated individually for its reported use in cancer in any community worldwide. The information regarding ethnopharmacological or folklore uses was searched in reputable journals and any data presented in the form of interviews, community surveys, or data collected from local inhabitants/herbal practitioners was extracted and analyzed.

\subsection{Exclusion Criteria}

Exclusion criteria included clinical trials reported for cancer using sources other than natural products; any clinical trial for cancer using natural products without proper ethnopharmacological relevance or community use in cancer; all natural products with a sound ethnopharmacological relevance in cancer but are yet to be evaluated in a clinical study; all preclinical studies (in vivo animal models or cell culture reports); any incomplete or duplicate study; clinical trials using vitamins, minerals, and conventional drugs only; and phase- 0 clinical trials.

Review period: An extensive search strategy was applied where retrospective data were collected without any restriction from September 2019 to April 2020. The literature was collected according to eligibility criteria, studied, and reported in the review herein. Until preparation and finalization of the manuscript, we updated the literature data on a regular basis, and any new information, if obtained, was added to the literature search.

Search result: The literature search consisted of 1342 articles, which was confined to 73 following a proper scrutiny of the eligible articles according to the pre-defined criteria. The flow diagram for selection and scrutiny of the literature is given in Figure 1. 

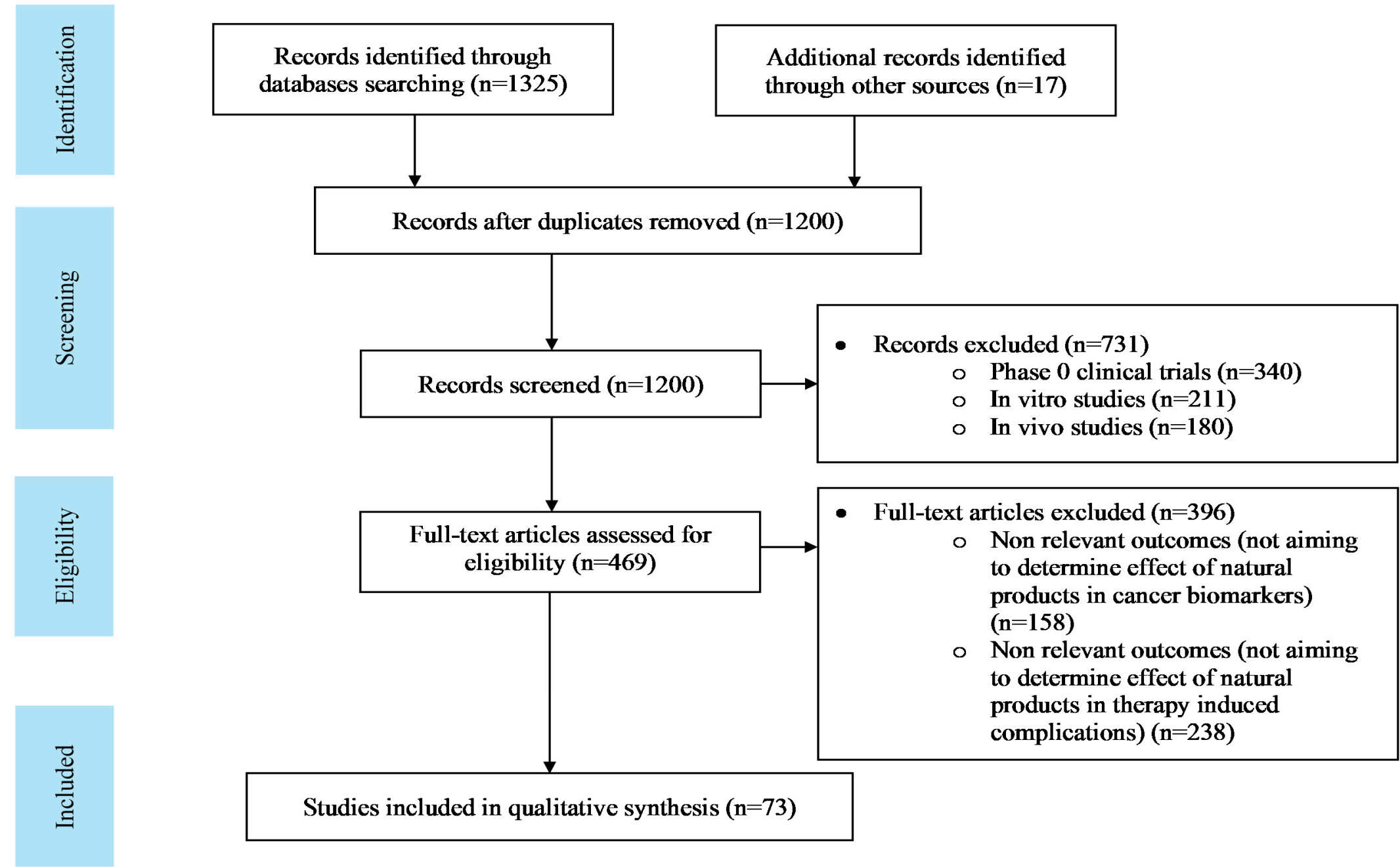

Figure 1. PRISMA (Preferred Reporting Items for Systematic Reviews and Meta-Analyses) flow sheet for literature search. 


\section{Literature Search}

The relevant literature data were studied and the extracted information is presented in a step-wise pattern below.

\subsection{Folklore Uses/Ethnopharmacological Relevance of the Selected Herbs in Cancer}

The ethnopharmacological relevance for the included herbs is given in detail in Table 1. The herbs were reported to have folklore uses in cancer in various communities including those of Pakistan, Palestine, Morocco, India, Turkey, Bangladesh, Ghana, Jordan, and Yemen. Various parts of these herbs such as leaves, fruit, dried sap, bulb, flowers, barks, rhizomes, seeds, rind, resin, and oleogum have been used for the intended purposes. Various types of cancers such as breast, head, skin, stomach, colorectal, liver, lungs, esophageal, and prostate cancers have been cured or treated using these herbs. These applications were used as a source of evidence for folklore or ethnopharmacological relevance of the selected herbs in order to evaluate the application and quality of clinical trials performed.

\subsection{Cancer Clinical Trials}

We searched the literature for clinical trials involving the selected herbs, finding a total of 73 clinical trials: aloe (15), black seeds (2), Boswellia (5), chamomile (3), colocynth (1), garlic (4), ginger (20), onion (1), pomegranate (8), senna (4), terebinth (1), thyme (3), wheat (5), and wormwood (1).

The current section highlights the key features of each clinical trial conducted for herbs in cancer. The part of an herbal product used as such or in a dosage form, the type of cancer studied, and the observed outcomes in a clinical trial were concluded as shown in Table 2.

\subsection{Evaluation of Clinical Trials (Jadad, Delphi, and Cochrane Scale)}

Three different scales, i.e., Jadad, Delphi, and Cochrane, were used in this study to evaluate clinical trials individually as per the items mentioned in the scales (Table 3). Each scale evaluated the quality of a clinical trial on certain specific key features mentioned in the table, where most of the items were overlapping/common for the three scales. Every scale possessed positive as well as negative aspects in terms of evaluation criteria and it is worth mentioning that no "best-fit scale" exists to critically analyze a clinical trial; hence, the three scales were applied together for assessing the quality of each clinical trial. This step may help cover the deficiencies present in a scale. In addition, as Jadad is the only scale that uses a scale (0-5) to assign score to a study, whereas Delphi and Cochrane lack a scoring system. For the sake of simplicity and ease of calculation/comparative scoring, we assigned an internal score to each item of the Delphi (0-9) and Cochrane scale (0-10). This summed up the total score for the three scales as being between 0 and 24 [18]. All the clinical trials included in this study were assessed and scored using the three scales and an individual as well as final score were assigned for classification of the clinical trials. Table 4 shows in detail all the items present in the three scales and the deficiencies observed for individual clinical trials according to the items in each scale. 
Table 1. Literature regarding ethnopharmacological relevance of the plants used in cancer.

\begin{tabular}{|c|c|c|c|}
\hline Herb & Botanical Name & Part/s Used & Ethnopharmacological Relevance \\
\hline Aloe & Aloe vera & $\begin{array}{l}\text { leaves, dried sap (fluid), } \\
\quad \text { extracted gel }\end{array}$ & breast cancer in Palestine [19] and lung cancer in a Pakistani community [20] \\
\hline Black seeds & Nigella sativa & seeds & general/colorectal cancer in Morocco [21] and Bangladesh [22] \\
\hline Boswellia & Boswellia serrata & bark & general cancers in Near East region [23] and India [24] \\
\hline Chamomile & Matricaria suaveolens koch & flowers & lung, liver, and prostate cancer in Palestine $[19,25]$ \\
\hline Colocynth & Citrullus lanatus & fruit & general cancer treatments in Pakistan [25] and India [24] \\
\hline Garlic & Allium sativum & bulb, leaves & $\begin{array}{c}\text { general cancers in Morocco [21] and treatment of lung, esophageal, and breast cancers } \\
\text { in Palestine [19] }\end{array}$ \\
\hline Ginger & Zingiber officinale & rhizome & general cancers in Morocco [21] and stomach cancer in Palestine [19] \\
\hline Onion & Allium cepa & leaves, bulb, oil and seeds & skin cancers in Ghana [26] and general cancers in Jordan [27] \\
\hline Pomegranate & Punica granatum & fruit, rind & general cancers in Yemen [28] and skin cancers in Morocco [21] \\
\hline Senna & Cassia senna & leaves & general cancer treatments in India $[29,30]$ \\
\hline Terebinth & Pistacia terebinthus & resin, branches, fruit & Islamic traditional medicine [31] and general cancer in Turkey [32] \\
\hline Thyme & Thymus vulgaris & leaves & head, lung, colorectal cancer in Turkey [33] and breast cancer in Palestine [34] \\
\hline Wheat & Triticum aestivum & shoot & general cancer [35] and breast/colorectal cancers in Trinidad [36] \\
\hline Wormwood & $\begin{array}{l}\text { Artemisia abaensis, } \\
\text { Artemisia abbreviata }\end{array}$ & leaves & general/digestive cancers in Turkey and Morocco $[21,37]$ \\
\hline
\end{tabular}

Table 2. Clinical trials in cancer for the selected herbs with details about type of cancer and its outcome observed.

\begin{tabular}{|c|c|c|c|c|}
\hline Plant & Trial & Part/Dosage Form Used & Type of Cancer & Results \\
\hline \multirow{12}{*}{ Aloe } & A1 & gel & breast cancer & $\downarrow$ radiation-induced skin side effects [38] \\
\hline & A2 & lotion & head/neck, breast cancers & $\downarrow$ intensity of radiation-induced dermatitis [39] \\
\hline & A3 & aloe and myrrh mixture & various cancers & induced a control of the neoplastic disease [40] \\
\hline & A4 & pure gel syrup & head and neck cancer & $\downarrow$ severity of radiation-induced mucositis [41] \\
\hline & A5 & gel + natural agents & head and neck cancer & no effect on mucositis [42] \\
\hline & A6 & gel & breast cancer & $\downarrow$ prevalence of radiation-induced dermatitis [43] \\
\hline & A7 & juice & head and neck cancer & lack of effects as adjunct to head and neck radiotherapy [10] \\
\hline & A8 & gel & breast cancer & no effect against radiation-induced dermatitis [44] \\
\hline & A9 & gel & breast cancer & $\downarrow$ acute skin reactions [45] \\
\hline & A10 & cream & breast cancer & protective role with $\downarrow$ radiation-induced dermatitis [46] \\
\hline & A11 & ointment & pelvic malignancies & improved proctitis and enhanced QOL [47] \\
\hline & A12 & gel & head/neck, abdomen tumors & protective effect to prevent skin reactions [48] \\
\hline
\end{tabular}


Table 2. Cont

\begin{tabular}{|c|c|c|c|c|}
\hline Plant & Trial & Part/Dosage Form Used & Type of Cancer & Results \\
\hline \multirow{3}{*}{ Aloe } & A13 & mouthwash solution & \multirow{3}{*}{$\begin{array}{c}\text { acute myeloid and } \\
\text { lymphocytic leukemia } \\
\text { head and neck cancer } \\
\text { breast cancer }\end{array}$} & $\downarrow$ intensity of stomatitis pain [49] \\
\hline & A14 & juice & & $\downarrow$ severity of radiation-induced mucositis [50] \\
\hline & A15 & leaf extract cream & & no effect on acute skin toxicity or symptom severity [51] \\
\hline \multirow{2}{*}{ Black seeds } & B1 & seeds & brain tumor & $\downarrow$ incidence of febrile neutropenia [52] \\
\hline & B2 & oil in soft gelatin capsule & lymphoblastic leukemia & $\downarrow$ methotrexate hepatotoxicity [53] \\
\hline \multirow{5}{*}{ Boswellia } & $\mathrm{C} 1$ & extract as tablet & glioblastoma multiform & $\downarrow$ radio-chemotherapy-induced cerebral edema [54] \\
\hline & $\mathrm{C} 2$ & extract as capsule & brain tumors & $\downarrow$ cerebral edema measured by MRI [55] \\
\hline & $\mathrm{C} 3$ & Boswellia as cream & breast cancer & $\downarrow$ erythema and skin superficial symptoms [56] \\
\hline & $\mathrm{C} 4$ & $\begin{array}{l}\text { spirulina-curcumin-Boswellia } \\
\text { mixture }\end{array}$ & benign thyroid nodules & $\downarrow$ size of benign thyroid nodules [57] \\
\hline & C5 & Boswellia serrata as OPERA & $\begin{array}{l}\text { breast, lung, prostate, } \\
\text { endometrial cancer }\end{array}$ & improved chemotherapy-induced peripheral neuropathy symptoms \\
\hline \multirow{3}{*}{ Chamomile } & D1 & flower infusion & gastric or colorectal cancer & $\downarrow$ oral mucositis [59] \\
\hline & D2 & & head and neck cancer & $\downarrow$ radiation-related dermatitis [60] \\
\hline & D3 & mouthwash & mucositis & no effect on 5-fluorouracil-induced mucositis [61] \\
\hline Colocynth & E1 & dry fruit extract oil & neuropathy & no improvement in peripheral neuropathy [62] \\
\hline \multirow{4}{*}{ Garlic } & F1 & extracts as capsule & hematological malignancy & no effect in febrile neutropenia [63] \\
\hline & $\mathrm{F} 2$ & extract as capsule & colorectal adenoma & $\downarrow$ adenomas and suppressed growth/proliferation [64] \\
\hline & F3 & extract as capsule & colon and liver cancer & improved NK cell activity [65] \\
\hline & $\mathrm{F} 4$ & $\begin{array}{l}\text { extract }+ \text { steam-distilled } \\
\text { garlic oil in a supplement }\end{array}$ & gastric cancer & $\downarrow$ mortality due to gastric cancer [66] \\
\hline \multirow{11}{*}{ Ginger } & G1 & extract as capsules & solid tumors & $\downarrow$ chemotherapy-induced nausea and vomiting [67] \\
\hline & G2 & ginger as capsules & chemotherapy & no effect on chemotherapy-induced nausea/vomiting [68] \\
\hline & G3 & extract + essential oil & thyroid cancer & effective to prevent salivary gland/thyroid cancer [69] \\
\hline & G4 & extract as capsule & lung, head/neck cancers & no effect on nausea due to cisplatin therapy [70] \\
\hline & G5 & ginger powder capsule & ovary and cervix cancers & no effect on nausea due to cisplatin therapy [71] \\
\hline & G6 & ginger powder capsule & bone sarcomas & effective in chemotherapy-induced nausea/vomiting [72] \\
\hline & G7 & extract as capsules & chemotherapy & no clear results mentioned [73] \\
\hline & G8 & powder capsules & breast cancer & $\downarrow$ frequency of nausea and vomiting [74] \\
\hline & G9 & powder capsules & breast cancer & $\downarrow$ frequency of nausea and vomiting [75] \\
\hline & G10 & essential oils & breast cancer & no effect in chemotherapy-induced nausea/vomiting [76] \\
\hline & G11 & powder capsules & breast cancer & no effect in chemotherapy-induced nausea/vomiting [77] \\
\hline
\end{tabular}


Table 2. Cont

\begin{tabular}{|c|c|c|c|c|}
\hline Plant & Trial & Part/Dosage Form Used & Type of Cancer & Results \\
\hline \multirow{9}{*}{ Ginger } & G12 & powder capsules & lung cancer & no effect in chemotherapy-induced nausea/vomiting [78] \\
\hline & G13 & powder capsules & breast cancer & no well-defined effect in nausea and vomiting [79] \\
\hline & G14 & $\begin{array}{l}\text { purified liquid extract in } \\
\text { capsule }\end{array}$ & $\begin{array}{l}\text { alimentary, breast, } \\
\text { genitourinary, lung tumors }\end{array}$ & $\downarrow$ severity of acute chemotherapy-induced nausea [80] \\
\hline & G15 & powder + yogurt & breast cancer & $\downarrow$ nausea severity and vomiting episodes [81] \\
\hline & G16 & extract as capsule & chemotherapy & no benefit in chemotherapy-induced nausea/vomiting [82] \\
\hline & G17 & powder capsules & $\begin{array}{l}\text { breast, bladder, lung, gastric, } \\
\text { and prostate cancers }\end{array}$ & $\downarrow$ nausea during chemotherapy [83] \\
\hline & G18 & extract as capsule & $\begin{array}{l}\text { breast, lymphoma, and } \\
\text { colon tumors }\end{array}$ & $\begin{array}{l}\text { enhances chemotherapy-induced nausea-related quality of life and less } \\
\text { cancer-related fatigue [84] }\end{array}$ \\
\hline & G19 & essential oil & $\begin{array}{l}\text { acute leukemia and } \\
\text { lymphomas, tumors }\end{array}$ & no significant decrease in nausea [85] \\
\hline & G20 & ginger moxibustion & $\begin{array}{l}\text { ovarian, cervical, } \\
\text { endometrial cancer }\end{array}$ & $\downarrow$ gastrointestinal tract reactions to chemotherapy [86] \\
\hline Onion & H1 & fresh raw onion & breast cancer & $\downarrow$ tumor markers in breast cancer [87] \\
\hline \multirow{8}{*}{ Pomegranate } & $\mathrm{I} 1$ & whole fruit powder & prostate cancer & $\downarrow$ prostate-specific antigen [88] \\
\hline & $\mathrm{I} 2$ & extract pills & prostate cancer & no effect on 8-hydroxy-20-deoxyguanosine levels [89] \\
\hline & I3 & extract pills & prostate cancer & $\uparrow$ PSA (prostate-specific antigen) doubling time [90] \\
\hline & $\mathrm{I} 4$ & liquid extract & prostate cancer & no effect on PSA doubling time (PSADT) [91] \\
\hline & I5 & liquid extract & colorectal cancer & colon tissue gene expression changed [92] \\
\hline & I6 & liquid extract & colorectal cancer & effect on specific colon tissue miRs [93] \\
\hline & I7 & juice & prostate cancer & $\downarrow$ proliferation marker (c-Myc) [94] \\
\hline & I8 & juice & prostate cancer & $\uparrow$ PSA doubling time, cell proliferation, and apoptosis [95] \\
\hline \multirow{4}{*}{ Senna } & $\mathrm{J} 1$ & sennosides as tablets & colon cancer & effective in bowel preparation for colon surgery [96] \\
\hline & $\mathrm{J} 2$ & syrup & $\begin{array}{l}\text { lung, breast, stomach, liver, } \\
\text { colon, prostate tumors }\end{array}$ & no efficacy of senna over lactulose in terminal cancer patients [97] \\
\hline & $\mathrm{J} 3$ & extract as tablet & $\begin{array}{l}\text { lung, breast, gastric, liver, } \\
\text { prostate tumors }\end{array}$ & no statistically significant difference in laxative action [98] \\
\hline & $\mathrm{J} 4$ & senna solution & colonic and rectal carcinoma & $\begin{array}{l}\text { colonic or rectal resection with senna is better than polyethylene glycol, } \\
\text { especially patients with stenosis [99] }\end{array}$ \\
\hline
\end{tabular}


Table 2. Cont.

\begin{tabular}{ccccc}
\hline Plant & Trial & Part/Dosage Form Used & Type of Cancer & Results \\
\hline Terebinth & K1 & extracted fruit oil as soap & colorectal cancer & safe use in the treatment of skin toxicity [100] \\
\hline Thyme & L1 & $\begin{array}{c}\text { sage } \\
\text { tea-thyme-peppermint } \\
\text { hydrosol } \\
\text { thyme honey } \\
\text { thyme honey }\end{array}$ & $\begin{array}{c}\text { colon, rectal, esophageal, } \\
\text { gastric, breast cancers } \\
\text { head and neck cancer } \\
\text { head and neck cancer }\end{array}$ & $\begin{array}{c}\text { oral mucositis [101] } \\
\text { colorectal cancer } \\
\text { L3 }\end{array}$ \\
effective in radiation-induced xerostomia [103]
\end{tabular}

Table 3. Scales for clinical trial evaluation and the items in each scale.

\begin{tabular}{|c|c|c|}
\hline Jadad Score Calculation & Final Delphi List & Cochrane Back Review Group List \\
\hline \multirow{2}{*}{$\begin{array}{l}\text { Was the study described as randomized (this includes words such as } \\
\text { randomly, random, and randomization)? } \\
\text { Was the method used to generate the sequence of randomization described } \\
\text { and appropriate (table of random numbers, computer generated, etc.)? }\end{array}$} & \multirow{2}{*}{$\begin{array}{l}\text { 1. Treatment allocation } \\
\text { (a) Was a method of randomization performed? } \\
\text { (b) Was the treatment allocation concealed? }\end{array}$} & Was the method of randomization adequate? \\
\hline & & Was the treatment allocation concealed? \\
\hline Was the study described as double blind? & $\begin{array}{l}\text { 2. Were the groups similar at baseline regarding } \\
\text { the most important prognostic indicators? }\end{array}$ & $\begin{array}{l}\text { Were the groups similar at baseline } \\
\text { regarding the most important } \\
\text { prognostic indicators? }\end{array}$ \\
\hline $\begin{array}{l}\text { Was the method of double blinding described and appropriate (identical } \\
\text { placebo, active placebo, dummy, etc.)? }\end{array}$ & 3. Were the eligibility criteria specified? & Was the patient blinded to the intervention? \\
\hline
\end{tabular}


Table 3. Cont

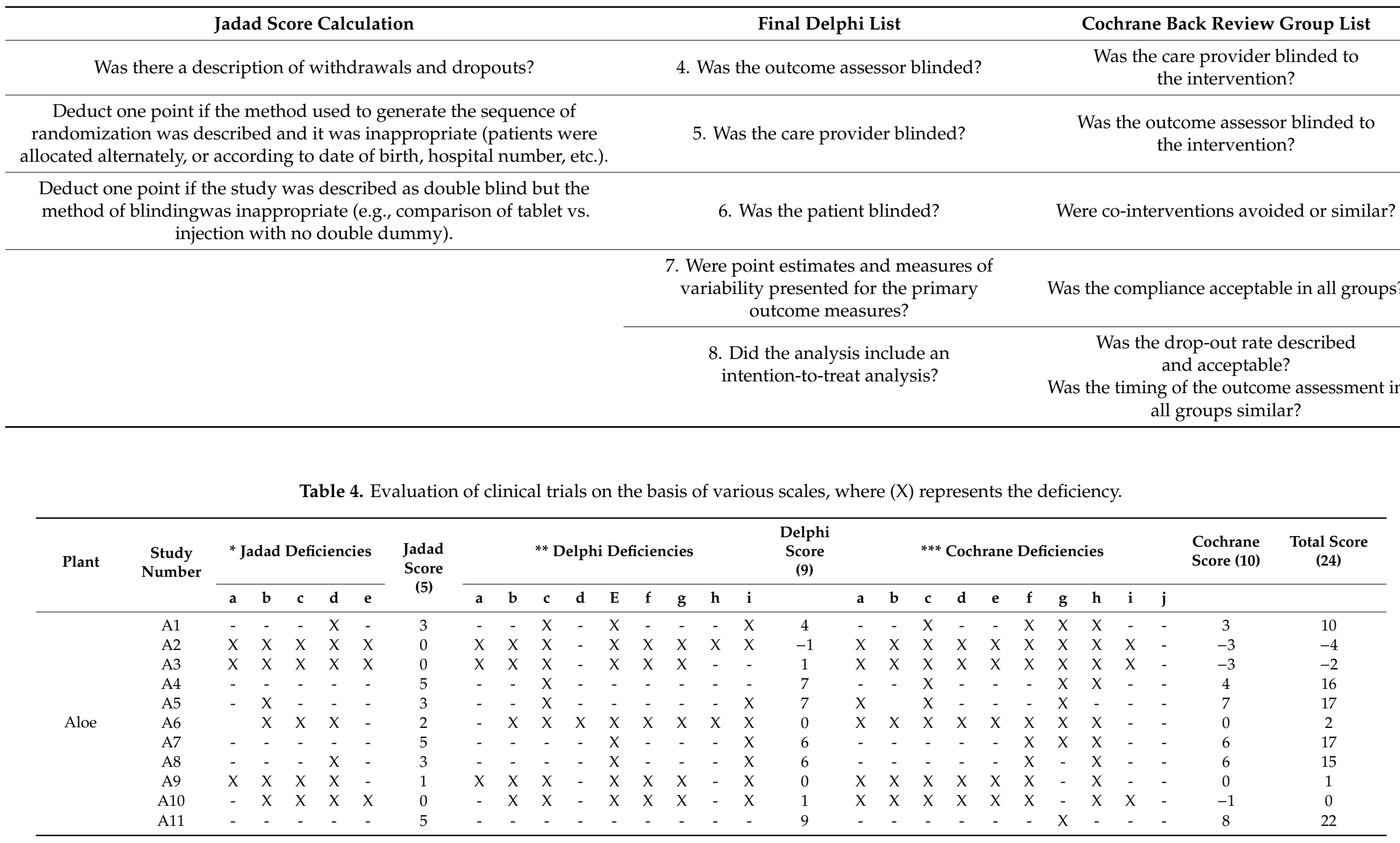


Table 4. Cont

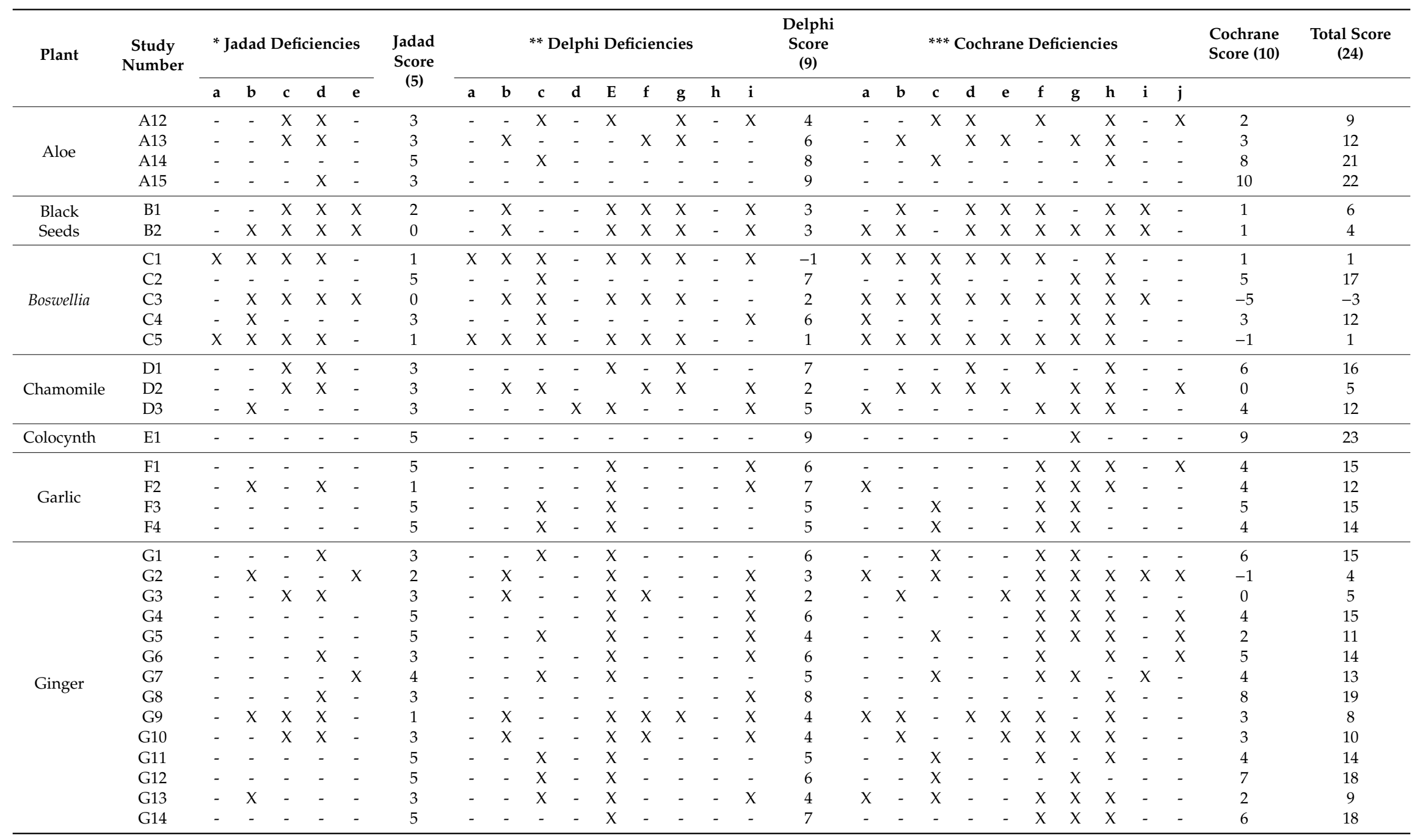


Table 4. Cont.

\begin{tabular}{|c|c|c|c|c|c|c|c|c|c|c|c|c|c|c|c|c|c|c|c|c|c|c|c|c|c|c|c|c|c|}
\hline \multirow{2}{*}{ Plant } & \multirow{2}{*}{$\begin{array}{l}\text { Study } \\
\text { Number }\end{array}$} & \multicolumn{5}{|c|}{ * Jadad Deficiencies } & \multirow{2}{*}{$\begin{array}{c}\text { Jadad } \\
\text { Score } \\
(5)\end{array}$} & \multicolumn{9}{|c|}{ ** Delphi Deficiencies } & \multirow{2}{*}{$\begin{array}{l}\text { Delphi } \\
\text { Score } \\
\text { (9) }\end{array}$} & \multicolumn{10}{|c|}{${ }^{* * *}$ Cochrane Deficiencies } & \multirow[t]{2}{*}{$\begin{array}{l}\text { Cochrane } \\
\text { Score (10) }\end{array}$} & \multirow[t]{2}{*}{$\begin{array}{c}\text { Total Score } \\
\text { (24) }\end{array}$} \\
\hline & & $\mathrm{a}$ & $\mathbf{b}$ & c & d & e & & $\mathrm{a}$ & $\mathbf{b}$ & c & $\mathrm{d}$ & $\mathrm{E}$ & $\mathrm{f}$ & $\mathrm{g}$ & h & i & & $\mathbf{a}$ & b & c & $\mathrm{d}$ & e & $f$ & $\mathrm{~g}$ & h & i & $\mathrm{j}$ & & \\
\hline \multirow{6}{*}{ Ginger } & G15 & - & $x$ & $x$ & $x$ & $x$ & 0 & - & $x$ & - & - & $x$ & $x$ & $x$ & - & - & 4 & $x$ & $x$ & - & $x$ & $x$ & $x$ & $x$ & $x$ & $x$ & - & 0 & 4 \\
\hline & G16 & - & - & - & $x$ & - & 3 & - & - & - & - & - & - & - & - & - & 9 & - & - & - & - & - & - & $x$ & $x$ & - & - & 8 & 20 \\
\hline & G17 & - & $x$ & $x$ & $x$ & - & 1 & - & $x$ & - & - & $x$ & $x$ & $x$ & - & $x$ & 3 & $x$ & $x$ & - & $x$ & $x$ & $x$ & $x$ & $x$ & - & - & 1 & 5 \\
\hline & G18 & - & - & - & - & - & 5 & - & - & - & - & - & - & - & - & - & 9 & - & - & - & - & - & - & $x$ & $x$ & - & - & 8 & 22 \\
\hline & G19 & - & - & - & $X$ & $x$ & 2 & - & - & - & - & $x$ & - & - & - & - & 7 & - & - & - & - & - & $x$ & $x$ & $x$ & $x$ & $x$ & 2 & 11 \\
\hline & G20 & - & - & $x$ & $x$ & - & 3 & - & $x$ & - & - & - & $x$ & $x$ & - & - & 6 & - & $x$ & - & $x$ & $x$ & - & - & - & - & - & 7 & 16 \\
\hline \multirow{8}{*}{ Pomegranate } & I1 & - & - & - & - & - & 5 & - & - & - & - & - & - & - & - & $x$ & 8 & - & - & - & - & - & - & $x$ & - & - & - & 8 & 21 \\
\hline & I2 & - & - & - & - & - & 5 & - & - & - & - & - & - & - & - & $x$ & 8 & - & - & - & - & - & - & $x$ & $x$ & - & - & 7 & 20 \\
\hline & I3 & - & - & - & $x$ & - & 3 & - & - & - & - & $x$ & - & - & - & - & 7 & - & - & - & - & - & $x$ & $x$ & $x$ & - & - & 6 & 16 \\
\hline & I4 & - & - & - & - & - & 5 & - & - & - & $x$ & & - & - & - & - & 8 & - & - & - & - & - & - & - & - & - & $x$ & 8 & 21 \\
\hline & I5 & - & $x$ & $x$ & $X$ & - & 1 & - & $x$ & - & - & $x$ & $x$ & $x$ & - & $x$ & 3 & $x$ & $x$ & & $x$ & $x$ & $x$ & $x$ & $x$ & - & $x$ & 0 & 4 \\
\hline & I6 & - & - & - & $X$ & - & 3 & - & - & - & - & $x$ & - & - & - & $x$ & 6 & - & - & - & - & - & $x$ & $x$ & $x$ & - & $x$ & 3 & 12 \\
\hline & I7 & - & $x$ & $x$ & $x$ & $x$ & 0 & - & $x$ & $x$ & - & $x$ & $x$ & $x$ & - & $x$ & 1 & $x$ & $x$ & $x$ & $x$ & $x$ & $x$ & $x$ & $x$ & $x$ & - & -2 & -1 \\
\hline & I8 & $x$ & $x$ & $x$ & $x$ & - & 1 & $x$ & $x$ & $x$ & - & - & $x$ & $x$ & - & $x$ & 2 & $x$ & $X$ & $x$ & $x$ & $x$ & - & - & $x$ & - & - & 2 & 5 \\
\hline \multirow{3}{*}{ Senna } & $\mathrm{J} 1$ & - & - & $x$ & $x$ & - & 3 & - & $x$ & - & $x$ & $x$ & $x$ & $x$ & - & - & 3 & - & $x$ & - & $x$ & $x$ & $x$ & - & $x$ & - & - & 3 & 9 \\
\hline & $\mathrm{J} 2$ & - & $x$ & $x$ & $x$ & - & 1 & - & $x$ & - & - & $x$ & $x$ & $x$ & - & $x$ & 3 & $x$ & $x$ & - & $x$ & $x$ & $x$ & $x$ & $x$ & - & - & 1 & 5 \\
\hline & $\mathrm{J} 4$ & - & - & $x$ & $x$ & - & 3 & - & - & - & - & $x$ & - & $x$ & - & $x$ & 5 & - & - & - & $x$ & - & $x$ & $x$ & $x$ & - & $x$ & 2 & 10 \\
\hline Terebinth & K1 & $x$ & $x$ & $x$ & $x$ & $x$ & 0 & $x$ & $x$ & $x$ & - & $x$ & $x$ & $x$ & $x$ & - & 0 & $x$ & $x$ & $x$ & $x$ & $x$ & $x$ & - & - & $x$ & - & 1 & 1 \\
\hline \multirow{3}{*}{ Thyme } & L1 & - & - & $x$ & $x$ & - & 3 & - & $x$ & - & - & $x$ & $x$ & $x$ & - & - & 4 & - & $x$ & - & $x$ & $x$ & $x$ & $x$ & - & - & - & 4 & 11 \\
\hline & L2 & - & - & $x$ & $x$ & - & 3 & - & $x$ & - & - & - & $x$ & $x$ & - & $x$ & 5 & - & $x$ & - & $x$ & $x$ & - & $x$ & $x$ & - & - & 3 & 11 \\
\hline & L3 & - & - & $x$ & $x$ & - & 3 & - & $x$ & - & - & - & $x$ & $x$ & - & $x$ & 5 & - & $x$ & - & $x$ & $x$ & - & $x$ & $x$ & - & - & 3 & 11 \\
\hline \multirow{5}{*}{ Wheat } & M1 & $x$ & $x$ & $x$ & $x$ & $x$ & 0 & $x$ & $x$ & $x$ & - & $x$ & $x$ & $x$ & - & $x$ & 1 & $x$ & $x$ & $x$ & $x$ & $x$ & $x$ & $x$ & $x$ & $x$ & - & -1 & 0 \\
\hline & M2 & - & $x$ & $x$ & $X$ & - & 1 & - & $x$ & - & - & $x$ & $x$ & $x$ & - & $x$ & 3 & $x$ & $x$ & - & $x$ & $x$ & $x$ & - & $x$ & - & - & 2 & 6 \\
\hline & M3 & $x$ & $x$ & $x$ & $X$ & $x$ & 0 & $x$ & $x$ & $X$ & - & $x$ & $x$ & - & - & - & 2 & $x$ & $x$ & $x$ & - & $x$ & $x$ & - & - & $x$ & - & 2 & 4 \\
\hline & M4 & $x$ & $x$ & $x$ & $x$ & $x$ & 0 & $x$ & $x$ & - & - & $x$ & $x$ & $x$ & - & - & 3 & $x$ & $x$ & - & $x$ & $x$ & $x$ & $x$ & $x$ & $x$ & - & 0 & 3 \\
\hline & M5 & - & $x$ & $x$ & $x$ & $x$ & 0 & - & $x$ & - & - & $x$ & $x$ & $x$ & - & - & 4 & $x$ & $x$ & - & $x$ & $x$ & $x$ & - & $x$ & $x$ & - & 2 & 6 \\
\hline Wormwood & N1 & $x$ & $x$ & $x$ & $x$ & - & 1 & $x$ & $x$ & $X$ & $x$ & $x$ & $x$ & $x$ & - & $x$ & -1 & $x$ & $x$ & $x$ & $x$ & $x$ & $x$ & $x$ & $x$ & - & - & -1 & -1 \\
\hline
\end{tabular}




\subsection{Statistical Analysis}

Statistical tools of PCA (principal component analysis) and Pearson's correlation were used to categorize the data. The factors analyzed showed a total variability of $68.21 \%$ for four components (PC1-PC4), as shown in Table 5. The scree plot for the components is presented in Figure 2. An individual variability (\%) was observed as PC1 (30.81), PC2 (14.70), PC3 (12.69), and PC4 (9.99). The factors loaded in PC1, i.e., the highest variability, were the deficiencies of non-blinding of the clinical trials, no concealment of the treatment allocation, and non-blindness of the patient and care provider. The next high percentage variability, i.e., PC2, showed loading for deficiencies, non-randomization of the studies, non-blinding of the outcome assessors, no proper drop-out procedures, and lack of information regarding baseline characteristics for the groups. The individual percentage variability with cumulative percentage is shown in Table 5. In addition, a three-dimensional representation of the deficiencies in the components is shown in Figure 2.

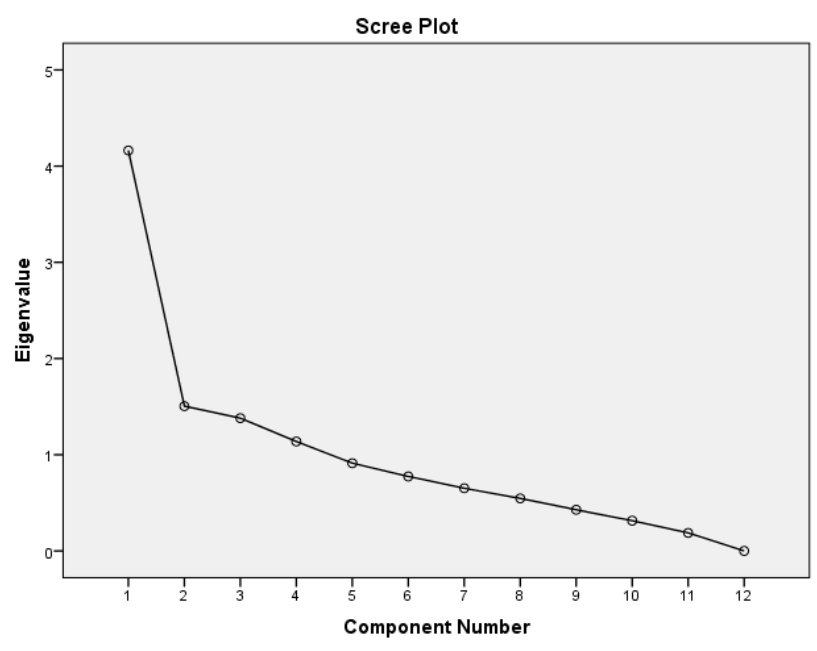

(a)

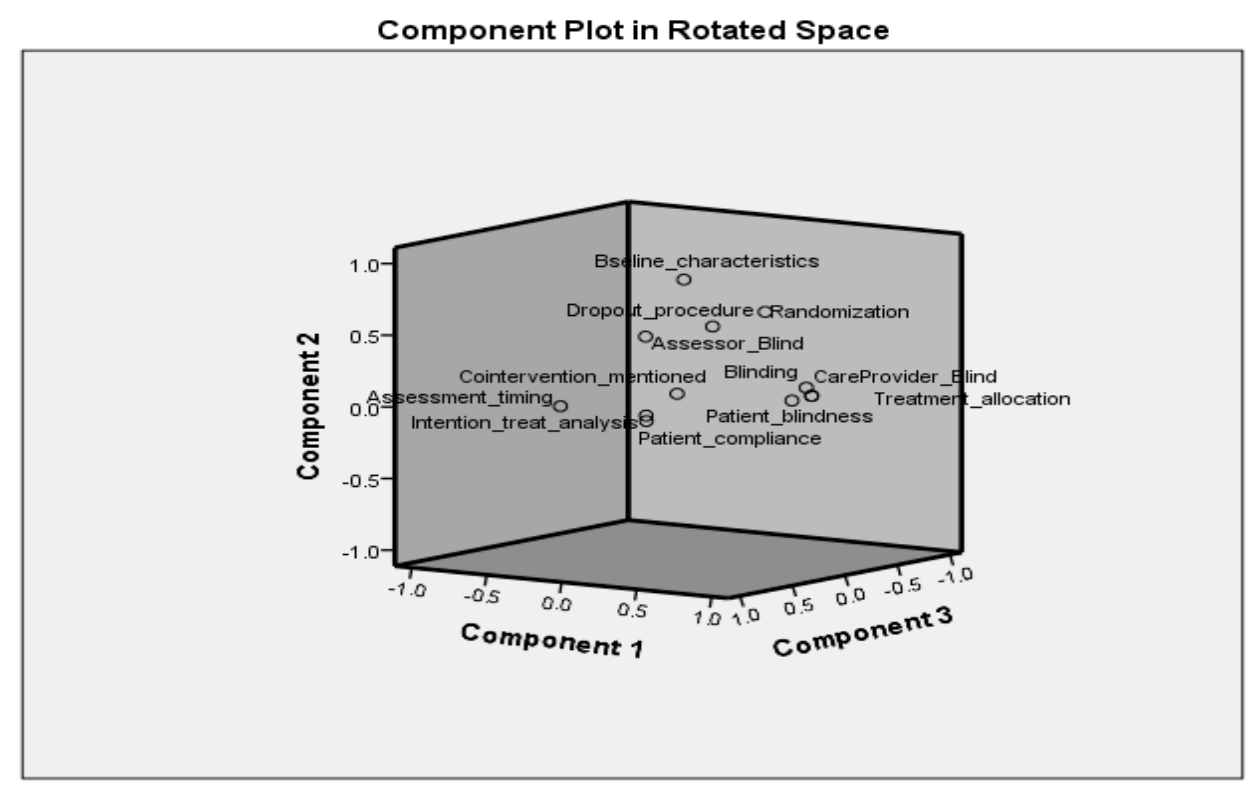

(b)

Figure 2. Scree plot (a) and loading (b) of components. 
Table 5. Principal components analysis (PCA) with component loading.

\begin{tabular}{lcccc}
\hline \multicolumn{1}{c}{ Factors } & PC1 & PC2 & PC3 & PC4 \\
\hline (A) Clinical trial randomized or non-randomized & 0.542 & 0.662 & -0.053 & 0.013 \\
(B) Clinical trial blinded or non-blinded & 0.792 & 0.153 & -0.088 & 0.121 \\
(C) Treatment allocation concealed or not & 0.930 & 0.131 & 0.058 & 0.010 \\
(D) The outcome assessor was blinded or non-blinded & 0.090 & 0.515 & 0.441 & -0.416 \\
$\begin{array}{l}\text { (E) Patient was blinded in the study or non-blinded } \\
\text { (F) The care provider was blinded or non-blinded }\end{array}$ & 0.871 & 0.107 & 0.162 & -0.067 \\
(G) The intention to treat analysis was mentioned in & 0.930 & 0.131 & 0.058 & 0.010 \\
clinical trial or not & 0.168 & -0.013 & 0.547 & 0.478 \\
(H) Proper drop-out procedure mentioned or not & 0.304 & 0.559 & 0.108 & -0.162 \\
(I) Patient compliance for the clinical trial reported or not & 0.269 & -0.020 & 0.689 & 0.061 \\
(J) Timing of outcome assessment mentioned or not & -0.29 & 0.028 & 0.700 & -0.092 \\
(K) Baseline characteristics for the group were & -0.03 & 0.822 & -0.100 & 0.215 \\
mentioned or not $\quad$ Variability \% & & & & \\
(L) Co-interventions were mentioned or not & 0.005 & 0.047 & 0.019 & 0.833 \\
$\quad 30.81$ & 14.70 & 12.69 & 9.99 \\
$\quad 30.81$ & 45.52 & 58.22 & 68.21 \\
\hline
\end{tabular}

Pearson's correlation was constructed to cross-verify the PCA analysis. For Pearson's correlation, all the pairs showed a positive correlation, i.e., none of the pairs were found to have a negative correlation. A similar phenomenon to PCA was observed in Pearson's correlation. Even for the deficiency "co-interventions were not mentioned" that was loaded separately in PC4 of the PCA was observed with no correlation/pair to any other factors in Pearson's analysis. The correlation matrix for Pearson's analysis is shown in detail in Table 6.

The statistical analysis below confirms a descending order (Figure 3) of occurrence for the deficiencies.

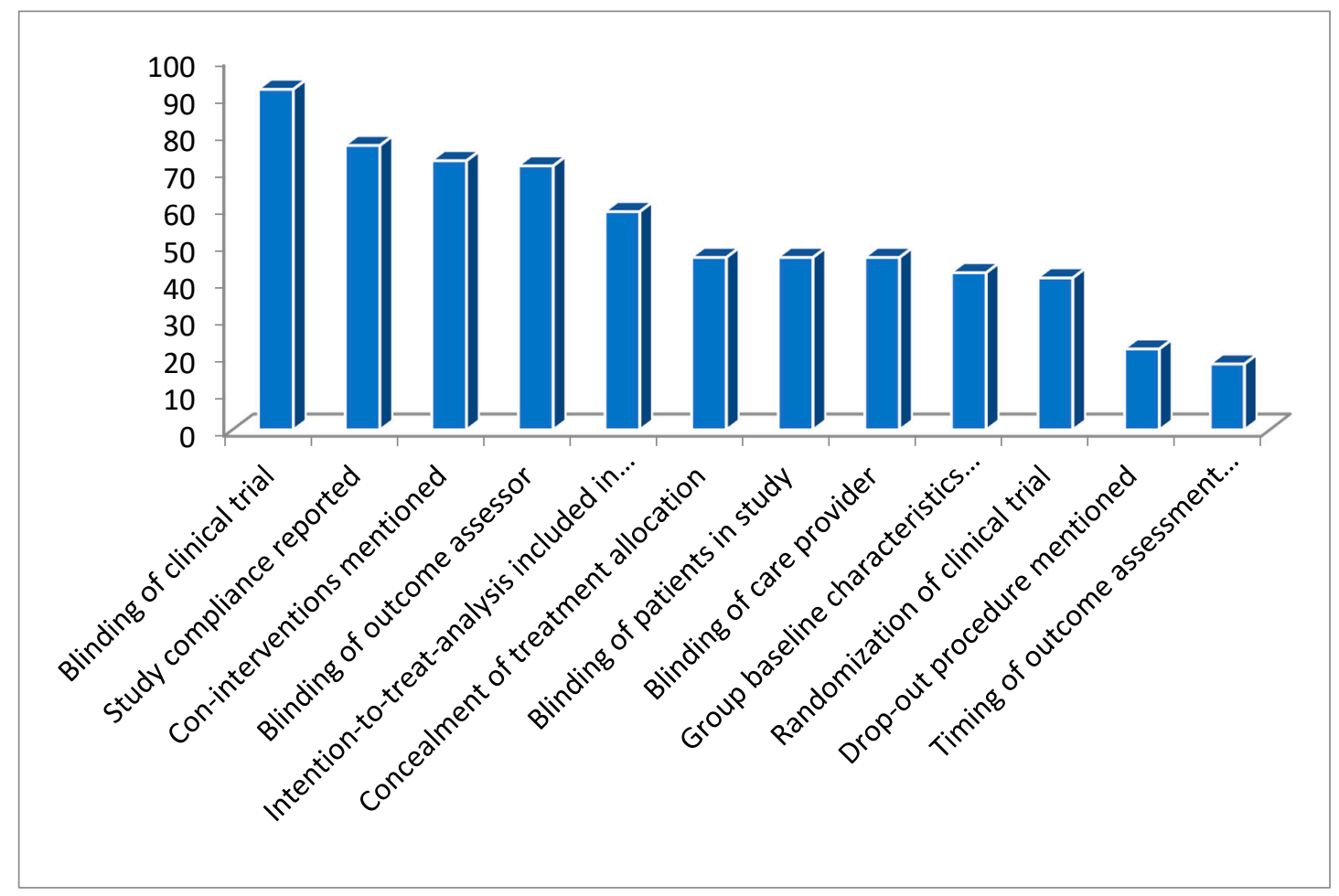

Figure 3. Figure representing the percentage of deficiencies in these clinical trials. 
Table 6. Pearson correlation analysis; A-L denotes factors mentioned in Table 5.

\begin{tabular}{|c|c|c|c|c|c|c|c|c|c|c|c|c|}
\hline & A & B & $\mathrm{C}$ & D & $E$ & $F$ & G & $\mathbf{H}$ & I & $\mathbf{J}$ & K & L \\
\hline A & 1 & & & & & & & & & & & \\
\hline B & 0.503 & 1 & & & & & & & & & & \\
\hline $\mathrm{C}$ & 0.54 & 0.626 & 1 & & & & & & & & & \\
\hline D & 0.31 & 0.035 & 0.204 & 1 & & & & & & & & \\
\hline $\mathrm{E}$ & 0.516 & 0.72 & 0.767 & 0.164 & 1 & & & & & & & \\
\hline $\mathbf{F}$ & 0.54 & 0.626 & 1 & 0.204 & 0.767 & 1 & & & & & & \\
\hline G & 0.155 & 0.13 & 0.172 & 0.065 & 0.166 & 0.172 & 1 & & & & & \\
\hline $\mathbf{H}$ & 0.426 & 0.299 & 0.335 & 0.31 & 0.307 & 0.335 & -0.059 & 1 & & & & \\
\hline I & 0.101 & 0.143 & 0.237 & 0.256 & 0.293 & 0.237 & 0.238 & 0.127 & 1 & & & \\
\hline $\mathbf{J}$ & -0.193 & -0.209 & -0.203 & 0.137 & -0.033 & -0.203 & 0.181 & 0.051 & 0.172 & 1 & & \\
\hline K & 0.453 & 0.151 & 0.102 & 0.187 & 0.101 & 0.102 & 0.037 & 0.184 & -0.018 & -0.009 & 1 & \\
\hline $\mathrm{L}$ & -0.036 & 0.07 & 0.053 & -0.16 & -0.058 & 0.053 & 0.145 & 0.051 & 0.093 & -0.049 & 0.055 & 1 \\
\hline
\end{tabular}


"non-blinding of clinical trials $>$ no compliance reported for the study $>$ co-interventions not mentioned $>$ outcome assessor non blinded > intention-to-treat analysis was not included in a study $>$ treatment allocation was not concealed $>$ patients were non-blinded $>$ care provider was non-blinded $>$ group baseline characteristics were not mentioned $>$ studies were non-randomized $>$ drop-out procedure were not mentioned $>$ timing for outcome assessment were not mentioned".

\subsection{Score for Clinical Trials}

An in-house grading scale was developed in order to simplify the classification of quality of the bulk of clinical trials. The clinical trials were graded out of 24 points, with distribution as "very poor quality" ( $\leq 6$ including negative values), "poor quality" (7 to 12$)$, "acceptable quality" (13 to 18$)$, "good quality" (19 to 24). Detailed information regarding study score is given in Table 7. A small portion, i.e., $16.44 \%$ (12 out of 73) of the studies were found to be of good quality, whereas more than half the proportion $(57.54 \% ; 27$ very poor +15 poor quality) of the studies were found to be of poor quality.

Table 7. Final in-house scoring for the clinical trials.

\begin{tabular}{cccc}
\hline Quality of Trial Based on Assigned Scale & $\begin{array}{c}\text { Frequency } \\
\mathbf{( N )}\end{array}$ & $\begin{array}{c}\text { Percent } \\
\mathbf{( \% )}\end{array}$ & Cumulative Percent (\%) \\
\hline $\begin{array}{c}\text { Very poor quality clinical trials } \\
\text { (6 and below, i.e., negative value) }\end{array}$ & 27 & 36.99 & 36.99 \\
\hline $\begin{array}{c}\text { Poor quality clinical trials } \\
(7-12)\end{array}$ & 15 & 20.55 & 57.53 \\
\hline $\begin{array}{c}\text { Acceptable quality clinical trials } \\
(13-18)\end{array}$ & 19 & 26.03 & 100.00 \\
\hline $\begin{array}{c}\text { Good quality clinical trials } \\
(19-24)\end{array}$ & 12 & 16.44 \\
\hline Total & 73 & 100.00 &
\end{tabular}

\section{Discussion}

The evaluation and assessment of the clinical trials developed a few basic questions necessary for any study such as "what is the source/background of the herb?", "what is its phytochemical profile?", "what are the parts, dosage forms, and extraction solvents used?", "what is the asking information, dose used, and its clinical phase studied?" All these questions are summarized briefly below.

Source and background data regarding the herb: Basic information relevant to the herb in terms of family, genus, species, folkloric use in cancer, geographical origin, and identification from authentic resources are very important. The plant may vary with regard to phytochemistry, which is subjected to differences in terms of place of origin, wherein altitude, temperature, stress, salinity, irrigation, etc. may affect the nature and quantity of active chemical present $[110,111]$. The majority of the trials were unable to explain the authentication process for the source of plant used and its background information. This may affect the quality of a clinical trial.

Phytochemical profile for the part used: The part of a plant may differ in the nature and amount of active chemicals when compared to other parts of the same plant; hence, there is a need for proper phytochemical profiling. These clinical trials used various parts of the plants such as leaves, roots, fruits, infusion, juice, and essential oils; however, the phytochemistry for the part of the plant used was missing in most of the studies [100].

Final dosage form used and its preparation/extraction: A number of clinical trials used dosage forms (extract or dried powder in capsule/tablet, creams, gels, mouthwashes, liquid extract, pills, syrup, etc.), however, the method of extraction or dosage form preparation was observed often. For a herbal product to be effective, appropriate extraction/processing is the basic step for success. The factors 
involved in extraction/drying/preparation of final dosage form (sunlight, temperature, solvent polarity and non-polarity, extraction time, pressure etc.) may either degrade or enhance the amount of an active ingredient or its activity thereof [112-114]. It is very crucial to investigate the effect of these factors upon the potential of an herbal dosage, but none of the clinical trial undertook such an investigation.

Choice of green solvent and extraction: Most of the extraction at present is performed using green solvents (water, ethanol, acetonitrile, etc.) due to dual properties of being eco- and human-friendly with less adverse effects. The infusion, juice, and fermentation products prepared in the reported clinical trials used alcohol-based solvents that are unhealthy, costly, and carry more adverse effects. Most of these solvents release toxic chemicals upon heating [115]. A need to shift to green extraction may be promoted.

Phase (0-V) selection: Although phase- 0 is mandatory for sub-therapeutic dose and toxicity, often the phase-I studies are skipped for the herbs with proper ethnopharmacological/folklore data available at community level [116]. In such cases, phase-II studies are started without a prior phase-I study, and hence proper evidence is necessary for an herb to start with a clinical trial.

Masking of clinical study: Equally important, a clinical trial should be properly blinded with respect to the patient, care provider/administrator, and data assessor in order to avoid the risk of bias in the data $[117,118]$. A number of clinical trials we reported do present the issue of improper blinding.

Treatment strategy (interactions, complications, and duration): The duration of treatment needs to be shorter in order to avoid complications, particularly in subjects using conventional medication for treatment. These clinical trials continued the studies from weeks until years, which is difficult at times because the subjects enrolled may either have stopped conventional medication or are already using natural products. Herbs are best known for their cytochrome P450 inhibitory or induction properties, of which both are dangerous. A long-term treatment strategy may expose the subjects to various herb-drug/drug-food interactions and nutritional deficiencies, which may produce emergency conditions. All these factors are the major sources of non-compliance in a study, being was observed in most of these clinical trials $[111,119,120]$.

Dose used: A dose up to $10 \mathrm{~g}$ in most of the cases was observed in these clinical trials. It is quite difficult to administer such a high dose in the form of a tablet or capsule as it outweighs the capacity for available size. In addition, it becomes impossible to administer a huge dose in divided doses, especially in subjects using conventional drugs where a serious risk of herb-drug interaction exists. More importantly for herbs with a lack of phase- 0 data, it is a serious risk to use such a high dose that can predispose potential health risks. This urges researchers to explore the herb for proper phase- 0 data, half-life, PKs (Pharmacokinetics), PDs (Pharmacodynamics), etc. and to ensure the quality variation for active principle in herbs, prior any clinical study [121,122]. The reported clinical trials did not mention any such information, which is utmost required for a study.

\section{Recommendations to Enhance Quality of a Clinical Trial}

Ethno-botanical/pharmacological and folklore evidence with quality evaluation: A detailed literature search needs to be ensured in order to collect appropriate information regarding the folklore use of a herb in various communities, followed by uniformity of geographical origin, part, family, genus, and species of the herb to study. In addition, a proper phytochemical profile must be established to evaluate and declare the quality and quantity of active chemicals present in a herb that are responsible for anticancer effects [117,118].

Herbal pharmacovigilance: Herbal pharmacovigilance is necessary to ensure the mainstream data necessary for a herbal clinical trial. Phase-0, i.e., toxicity studies, sub-therapeutic dose selection, adverse effects of the herb/herbal product, long-term effects, and herb-drug/food interaction studied, as well as PKs and PDs for half-life, metabolizing enzymes, and the excretion process need to be established. Pharmacovigilance assures a small dose with shorter treatment strategy/duration so as to avoid the untoward effects of the herb, especially when combined with conventional drugs. In addition, the pharmacovigilance ensures the quality variation and standardization of herbs [121,122]. 
Need for extraction or isolation and shape of final dosage form: The researcher needs to be clear regarding the pros and cons related to extracts/extraction and isolation. It is tiresome to isolate an active chemical; however, extracts due to presence of multi component nature pose restriction and complications when it is needed to study the molecular or genetic level effects for a treatment. Pursuit of isolation of the main active ingredient responsible for the cancerous effects in a herbal product/extract is mainly favored. In addition, dosage form compatibility is more important. Powder drugs and injectables are more easily absorbed and show enhanced bioavailability and therapeutic effects; still, the idea of nanoformulations, i.e., nano-particles, emulsions, micelles, and gels are more preferred due to low dose, enhanced and targeted treatment, and less side effects or adverse effects.

Clinical trials with tailored treatment approach: The paradigm shift from empirical to tailored approach, i.e., treatment strategy based on biologically relevant question, is the upcoming future for cancer clinical trials. Although the idea demands a profound change in infrastructure and methodology of clinical research and is challenging, it will bring about new opportunities in cancer treatment with a better understanding of the disease and mechanism of action of the treating agent [123]. The clinical trials may focus on acquiring such concept.

Immune-oncology: Clinical trials with a specific focus on immune boosting properties are also a unique source of accelerating cancer treatment [124]. A number of herbs such as leaves of muicle, aguacate, and muerdago; bark of cuachalalate and una de gato; and roots of matarique and guizazo de caballo have immune-enhancement/stimulant properties that may serve as a novel source of cancer treatment [125].

Nano-oncology: Nano-dosage form in the shape of liposomes, dendrimers, gold nanoparticles, micelles, nanoemulsions, nanogels, etc. are widely used in cancer treatment as they are inert, noncorrosive, targeted, safe, and free of the adverse effects associated with conventional treatments. A number of nano-dosage forms such as Myocet and Doxil for doxorubicin are available in the market [126]. It is worthwhile to convert the herbal products or extracts into various nano-dosage forms, which may add the benefits of more therapeutic efficiency and less adverse effects.

Precision medicine: Avoiding the idea of "one-size-fits-all" and matching the most appropriate and relevant individualized treatment approaches for a patient on the basis of the genetic profile of the patient and cancer type is known as precision medicine [127] In spite of tumor heterogeneity, which may affect precision medicine, promising outcomes may be observed if precision medicine is applied in herbal clinical trials.

\section{Conclusions}

The clinical trials in the systemic review revealed a poor quality according to the evaluation scales used. The majority of the studies were non-blinded and non-randomized. With respect to herbs used, a proper pharmacovigilance background was not reported in the studies. It is highly recommended that researchers enhance/uplift the studies of these clinical trials via addition of appropriate ethnopharmacological relevance, quality variation and standardization, phytochemical profile, focus on the hot area of cancer, and precision medicine when planning to conduct a clinical trial using a herb (powder/extract, etc.) or herbal product.

Author Contributions: R.A. and L.H.A. conceived the idea, study design, methodology, statistical analysis, discussion, and conclusion; L.H.A., A.K.A. and S.M.A. was responsible for the literature review, data extraction, introduction write up, evaluation, and scoring of individual clinical trial with tables for ethnopharmacological relevance and deficiencies/scoring of the clinical trials, as well as arrangement of the references. R.A. conducted analyses. All authors have read and agreed to the published version of the manuscript.

Funding: The study declares no funding from any governmental/private organizations.

Acknowledgments: No acknowledgment is applicable in this study.

Conflicts of Interest: No conflict of interest exists among the authors. 


\section{References}

1. National Cancer Institute. What Is Cancer? Differences between Cancer Cells and Normal Cells. National Cancer Institute. 2015. Available online: https://www.cancer.gov/about-cancer/understanding/what-is-cancer (accessed on 30 March 2020).

2. Fan, A.M. Information Resources in Toxicology. Cancer 2009, 1, 103-121.

3. Lyman, G.H. Risk factors for cancer. Prim Care 1992, 19, 465-479. [PubMed]

4. National Cancer Institute. Statistics at a Glance: The Burden of Cancer in the United States. Available online: https://www.cancer.gov/about-cancer/understanding/statistics (accessed on 30 March 2020).

5. Houts, P.S.; Lenhard, R.E.; Varricchio, C. ACS cancer facts and figures. Cancer Pract. 2019, 8, $105-108$.

6. Bray, F.; Ren, J.; Masuyer, E.; Ferlay, J. Global estimates of cancer prevalence for 27 sites in the adult population in 2008. Int. J. Cancer 2013, 132, 1133-1145. [CrossRef] [PubMed]

7. Orloff, G.M.; Champana, N.C.B. Cancer Treatments. Available online: https://winshipcancer.emory.edu/ research/index.html (accessed on 30 March 2020).

8. Rehman, S. An Overview of Cancer Treatment Modalities; IntechOpen: London, UK, 2018.

9. Shaikh, A.M.; Shrivastava, B.; Apte, K.G.; Navale, S.D. Medicinal plants as potential source of anticancer agents: A review. J. Pharm. Phytochem. 2016, 5, 291.

10. Su, C.K.; Mehta, V.; Ravikumar, L.; Shah, R.; Pinto, H.; Halpern, J.; Koong, A.; Goffinet, D.; Le, Q.-T. Phase II double-blind randomized study comparing oral aloe vera versus placebo to prevent radiation-related mucositis in patients with head-and-neck neoplasms. Int. J. Radiat. Oncol. Biol. Phys. 2004, 60, 171-177. [CrossRef] [PubMed]

11. Malik, A.; Afaq, F.; Sarfaraz, S.; Adhami, V.M.; Syed, D.N.; Mukhtar, H. Pomegranate fruit juice for chemoprevention and chemotherapy of prostate cancer. Proc. Natl. Acad. Sci. USA 2005, 102, 14813-14818. [CrossRef]

12. Shukla, Y.; Singh, M. Cancer preventive properties of ginger: A brief review. Food Chem. Toxicol. 2007, 45, 683-690. [CrossRef]

13. Srivastava, J.K.; Shankar, E.; Gupta, S. Chamomile: A herbal medicine of the past with a bright future. Mol. Med. Rep. 2010, 3, 895-901.

14. Nirmala, M.J.; Samundeeswari, A.; Sankar, P.D. Natural plant resources in anti-cancer therapy-A review. Res. Plant. Biol. 2011, 1, 1-14.

15. Alamgir, M.; Uddin, S.J. Recent advances on the ethnomedicinal plants as immunomodulatory agents. Ethnomed. Source Complement. Ther. 2010, 37, 227-244.

16. Ali-Shtayeh, M.S.; Jamous, R.M. Herbal medicines in cancer care in the Palestinian Authority. Eur. J. Integr. Med. 2011, 3, e129-e130. [CrossRef]

17. Getz, K. Protocol Design Trends and their Effect on Clinical Trial Performance. Available online: https://www.semanticscholar.org/paper/Protocol-Design-Trends-and-their-Effect-on-Clinical-Getz/ 52f088d59899d5b6cd5160fe0d916a92209ada6e (accessed on 30 March 2020).

18. Berger, V.W.; Alperson, S.Y. A general framework for the evaluation of clinical trial quality. Rev. Recent Clin. Trials 2009, 4, 79-88. [CrossRef] [PubMed]

19. Jaradat, N.A.; Al-Ramahi, R.; Zaid, A.N.; Ayesh, O.I.; Eid, A.M. Ethnopharmacological survey of herbal remedies used for treatment of various types of cancer and their methods of preparations in the West Bank-Palestine. BMC Complement. Altern. Med. 2016, 16, 93. [CrossRef] [PubMed]

20. Qadir, M.I. Medicinal and cosmetological importance of Aloe vera. Int. J. Nat. Ther. 2009, 2, 21-26.

21. Kabbaj, F.; Meddah, B.; Cherrah, Y.; Faouzi, E. Ethnopharmacological profile of traditional plants used in Morocco by cancer patients as herbal therapeutics. Phytopharmacology 2012, 2, 243-256.

22. Costa-Lotufo, L.V.; Khan, M.T.H.; Ather, A.; Wilke, D.V.; Jimenez, P.C.; Pessoa, C.; De Moraes, M.E.A.; De Moraes, M.O. Studies of the anticancer potential of plants used in Bangladeshi folk medicine. J. Ethnopharmacol. 2005, 99, 21-30. [CrossRef]

23. Abu-Darwish, M.S.; Efferth, T. Medicinal Plants from Near East for Cancer Therapy. Front. Pharmacol. 2018, 9, 56. [CrossRef]

24. Hussain, A.I.; Rathore, H.A.; Sattar, M.Z.A.; Chatha, S.A.S.; Sarker, S.D.; Gilani, A.H. Citrullus colocynthis (L.) Schrad (bitter apple fruit): A review of its phytochemistry, pharmacology, traditional uses and nutritional potential. J. Ethnopharmacol. 2014, 155, 54-66. [CrossRef] 
25. Memon, A.H.; Rind, F.M.A.; Laghari, M.G.H.; Mughal, U.R.; Memon, N.; Almani, F.; Khuhawar, M.Y. Study of folk recipes and ethnomedicinal uses of thirty medicinal plants of district Umerkot, Sindh, Pakistan. Hamdard Med. 2010, 53, 127-135.

26. Agyare, C.; Spiegler, V.; Asase, A.; Scholz, M.; Hempel, G.; Hensel, A. An ethnopharmacological survey of medicinal plants traditionally used for cancer treatment in the Ashanti region, Ghana. J. Ethnopharmacol. 2018, 212, 137-152. [CrossRef] [PubMed]

27. Aburjai, T.; Hudaib, M.; Tayyem, R.; Yousef, M.; Qishawi, M. Ethnopharmacological survey of medicinal herbs in Jordan, the Ajloun Heights region. J. Ethnopharmacol. 2007, 110, 294-304. [CrossRef] [PubMed]

28. Mothana, R.A.A.; Gruenert, R.; Lindequist, U.; Bednarski, P.J. Study of the anticancer potential of Yemeni plants used in folk medicine. Die Pharm.-An. Int. J. Pharm. Sci. 2007, 62, 305-307.

29. Begum, I.; Sharma, R.; Sharma, H.K. A Review on Plants Having Anti-Cancer Activity. Curr. Trends Pharm. Res. 2017, 4, 39-62.

30. Samant, S.S.; Vidyarthi, S.; Pant, S.; Sharma, P.; Marpa, S.; Sharma, P. Diversity, distribution, indigenous uses and conservation of the medicinal plants of Indian Himalayan region used in cancer. J. Biodivers. 2011, 2, 117-125. [CrossRef]

31. Javadi, B.; Iranshahy, M.; Emami, S.A. Anticancer plants in Islamic traditional medicine. Complement. Ther. Body Mind Soul 2015, 119. [CrossRef]

32. Polat, R.; Cakilcioglu, U.; Satıl, F. Traditional uses of medicinal plants in Solhan (Bingöl-Turkey). J. Ethnopharmacol. 2013, 148, 951-963. [CrossRef]

33. Algier, L.A.; Hanoglu, Z.; Özden, G.; Kara, F. The use of complementary and alternative (non-conventional) medicine in cancer patients in Turkey. Eur. J. Oncol. Nurs. 2005, 9, 138-146. [CrossRef]

34. Jaradat, N.A.; Shawahna, R.; Eid, A.M.; Al-Ramahi, R.; Asma, M.K.; Zaid, A.N. Herbal remedies use by breast cancer patients in the West Bank of Palestine. J. Ethnopharmacol. 2016, 178, 1-8. [CrossRef]

35. Zaid, H.; Rayan, A.; Said, O.; Saad, B. Cancer treatment by Greco-Arab and Islamic herbal medicine. Open Nutraceuticals J. 2010, 3, 203-212. [CrossRef]

36. Clement, Y.N.; Mahase, V.; Jagroop, A.; Kissoon, K.; Maharaj, A.; Mathura, P.; Mc Quan, C.; Ramadhin, D.; Mohammed, C. Herbal remedies and functional foods used by cancer patients attending specialty oncology clinics in Trinidad. BMC Complement. Altern. Med. 2016, 16, 399. [CrossRef] [PubMed]

37. Tas, F.; Ustuner, Z.; Can, G.; Eralp, Y.; Camlica, H.; Basaran, M.; Karagol, H.; Sakar, B.; Dişçi, R.; Topuz, E. The prevalence and determinants of the use of complementary and alternative medicine in adult Turkish cancer patients. Acta Oncol. 2005, 44, 161-167. [CrossRef] [PubMed]

38. Heggie, S.; Bryant, G.P.; Tripcony, L.; Keller, J.; Rose, P.; Glendenning, M.; Heath, J. A phase III study on the efficacy of topical aloe vera gel on irradiated breast tissue. Cancer Nurs. 2002, 25, 442-451. [CrossRef] [PubMed]

39. Haddad, P.; Amouzgar-Hashemi, F.; Samsami, S.; Chinichian, S.; Oghabian, M.A. Aloe vera for prevention of radiation-induced dermatitis: A self-controlled clinical trial. Curr. Oncol. 2013, 20, e345. [CrossRef]

40. Lissoni, P.; Rovelli, F.; Messina, G.; Brivio, F.; Boniardi, B.; Porro, G.; Vigore, L.; Di Fede, G.; Marchiori, P.; Brera, G. Biotherapy with the pineal hormone melatonin plus aloe and myrrh tincture in untreatable metastatic cancer patients as an essence therapy of cancer. Cancer Ther. 2009, 7, 397-401.

41. Sahebjamee, M.; Mansourian, A.; Hajimirzamohammad, M.; Zadeh, M.T.; Bekhradi, R.; Kazemian, A.; Manifar, S.; Ashnagar, S.; Doroudgar, K. Comparative Efficacy of Aloe vera and Benzydamine Mouthwashes on Radiation-induced Oral Mucositis: A Triple-blind, Randomised, Controlled Clinical Trial. Oral Health Prev. Dent. 2015, 13, 309-315.

42. Marucci, L.; Farneti, A.; Di Ridolfi, P.; Pinnarò, P.; Pellini, R.; Giannarelli, D.; Vici, P.; Conte, M.; Landoni, V.; Sanguineti, G. Double-blind randomized phase III study comparing a mixture of natural agents versus placebo in the prevention of acute mucositis during chemoradiotherapy for head and neck cancer. Head Neck 2017, 39, 1761-1769. [CrossRef]

43. Ahmadloo, N.; Kadkhodaei, B.; Omidvari, S.; Mosalaei, A.; Ansari, M.; Nasrollahi, H.; Hamedi, S.H.; Mohammadianpanah, M. Lack of prophylactic effects of aloe vera gel on radiation induced dermatitis in breast cancer patients. Asian Pac. J. Cancer Prev. 2017, 18, 1139.

44. Williams, M.S.; Burk, M.; Loprinzi, C.L.; Hill, M.; Schomberg, P.J.; Nearhood, K.; O'Fallon, J.R.; Laurie, J.A.; Shanahan, T.G.; Moore, R.L.; et al. Phase III double-blind evaluation of an aloe vera gel as a prophylactic agent for radiation-induced skin toxicity. Int. J. Radiat. Oncol. Biol. Phys. 1996, 36, 345-349. [CrossRef] 
45. Dudek, D.J.; Thompson, J.; Meegan, M.M.; Haycocks, T.R.; Barbieri, C.; Manchul, L.A. Pilot study to investigate the toxicity of Aloe vera gel in the management of radiation induced skin reactions for post-operative primary breast cancer. J. Radiother. Pract. 2000, 1, 197-204. [CrossRef]

46. Di Franco, R.; Sammarco, E.; Calvanese, M.G.; De Natale, F.; Falivene, S.; DiLecce, A.; Giugliano, F.M.; Murino, P.; Manzo, R.; Cappabianca, S.; et al. Preventing the acute skin side effects in patients treated with radiotherapy for breast cancer: The use of corneometry in order to evaluate the protective effect of moisturizing creams. Radiat. Oncol. 2013, 8, 57. [CrossRef] [PubMed]

47. Sahebnasagh, A.; Ghasemi, A.; Akbari, J.; Alipour, A.; Lashkardoost, H.; Ala, S.; Salehifar, E. Successful Treatment of Acute Radiation Proctitis with Aloe Vera: A Preliminary Randomized Controlled Clinical Trial. J. Altern. Complement. Med. 2017, 23, 858-865. [CrossRef]

48. Olsen, D.L.; Raub, W.; Bradley, C.; Johnson, M.; Macias, J.L.; Love, V.; Markoe, A. The effect of aloe vera $\mathrm{gel} / \mathrm{mild}$ soap versus mild soap alone in preventing skin reactions in patients undergoing radiation therapy. Oncol. Nurs. Forum 2001, 28, 543-547.

49. Mansouri, P.; Haghighi, M.; Beheshtipour, N.; Ramzi, M. The effect of aloe vera solution on chemotherapy-induced stomatitis in clients with lymphoma and leukemia: A Randomized controlled clinical trial. Int. J. Community Based Nurs. Midwifery 2016, 4, 119-126.

50. Puataweepong, P.; Dhanachai, M.; Dangprasert, S.; Sithatani, C.; Sawangsilp, T.; Narkwong, L.; Puttikaran, P.; Intragumtornchai, $\mathrm{T}$. The efficacy of oral Aloe vera juice for radiation induced mucositis in head and neck cancer patients: A double-blind placebo-controlled study. Asian Biomed. 2010, 3, 375-382.

51. Hoopfer, D.; Holloway, C.; Gabos, Z.; Alidrisi, M.; Chafe, S.; Krause, B.; Lees, A.; Mehta, N.; Tankel, K.; Strickland, F.; et al. Three-Arm Randomized Phase III Trial: Quality Aloe and Placebo Cream Versus Powder as Skin Treatment during Breast Cancer Radiation Therapy. Clin. Breast Cancer 2015, 15, 181-190.e4. [CrossRef] [PubMed]

52. Mousa, H.F.M.; Abd-El-Fatah, N.K.; Darwish, O.A.-H.; Shehata, S.F.; Fadel, S.H. Effect of Nigella sativa seed administration on prevention of febrile neutropenia during chemotherapy among children with brain tumors. Childs Nerv. Syst. 2017, 33, 793-800. [CrossRef] [PubMed]

53. Hagag, A.; Abdelaal, A.; Elfaragy, M.; Hassan, S.; Elzamarany, E. Therapeutic value of black seed oil in methotrexate hepatotoxicity in Egyptian children with acute lymphoblastic leukemia. Infect. Disord. Drug Targets 2015, 15, 64-71. [CrossRef]

54. Di, F.P.; Simonetti, G.; Petruzzi, A.; Bertuccioli, A.; Botta, L.; Bruzzone, M.G.; Cuccarini, V.; Fariselli, L.; Lamperti, E. A novel lecithin-based delivery form of Boswellic acids as complementary treatment of radiochemotherapy-induced cerebral edema in patients with glioblastoma multiforme: A longitudinal pilot experience. J. Neurosurg. Sci. 2019, 63, 286-291.

55. Kirste, S.; Treier, M.; Wehrle, S.J.; Becker, G.; Abdel-Tawab, M.; Gerbeth, K.; Hug, M.J.; Lubrich, B.; Grosu, A.L.; Momm, F. Boswellia serrata acts on cerebral edema in patients irradiated for brain tumors. Cancer 2011, 117, 3788-3795. [CrossRef]

56. Togni, S.; Maramaldi, G.; Bonetta, A.; Giacomelli, L.; Di Pierro, F. Clinical evaluation of safety and efficacy of Boswellia-based cream for prevention of adjuvant radiotherapy skin damage in mammary carcinoma: A randomized placebo controlled trial. Eur. Rev. Med. Pharmacol. Sci. 2015, 19, 1338-1344. [PubMed]

57. Stancioiu, F.; Mihai, D.; Papadakis, G.Z.; Tsatsakis, A.; Spandidos, D.A.; Badiu, C. Treatment for benign thyroid nodules with a combination of natural extracts. Mol. Med. Reports 2019, 20, 2332-2338. [CrossRef] [PubMed]

58. Desideri, I.; Francolini, G.; Becherini, C.; Terziani, F.; Paoli, C.D.; Olmetto, E.; Loi, M.; Perna, M.; Meattini, I.; Scotti, V.; et al. Use of an alpha lipoic, methylsulfonylmethane and bromelain dietary supplement (Opera ${ }^{\circledR}$ ) for chemotherapy-induced peripheral neuropathy management, a prospective study. Med. Oncol. 2017, 34, 46. [CrossRef] [PubMed]

59. dos Reis, P.E.D.; Ciol, M.A.; de Melo, N.S.; de Souza Figueiredo, P.T.; Leite, A.F.; de Melo Manzi, N. Chamomile infusion cryotherapy to prevent oral mucositis induced by chemotherapy: A pilot study. Support. Care Cancer 2016, 24, 4393-4398. [CrossRef]

60. Ferreira, E.B.; Ciol, M.A.; Vasques, C.I.; Bontempo, P.D.; Vieira, N.N.; Silva, L.F.; Avelino, S.R.; dos Santos, M.A.; dos Reis, P.E. Gel of chamomile vs, urea cream to prevent acute radiation dermatitis in patients with head and neck cancer: A randomized controlled trial. J. Adv. Nurs. 2016, 72, 1926-1934. [CrossRef] 
61. Fidler, P.; Loprinzi, C.L.; O’Fallon, J.R.; Leitch, J.M.; Lee, J.K.; Hayes, D.L.; Novotny, P.; Clemens-Schutjer, D.; Bartel, J.; Michalak, J.C. Prospective evaluation of a chamomile mouthwash for prevention of 5-FU-induced oral mucositis. Cancer 1996, 77, 522-525. [CrossRef]

62. Rostami, N.; Mosavat, S.H.; Heydarirad, G.; Arbab Tafti, R.; Heydari, M. Efficacy of topical Citrullus colocynthis (bitter apple) extract oil in chemotherapy-induced peripheral neuropathy: A pilot double-blind randomized placebo-controlled clinical trial. Phytother. Res. 2019, 33, 2685-2691. [CrossRef]

63. Gatt, M.E.; Strahilevitz, J.; Sharon, N.; Lavie, D.; Goldschmidt, N.; Kalish, Y.; Gural, A.; Paltiel, O.B. A Randomized Controlled Study to Determine the Efficacy of Garlic Compounds in Patients with Hematological Malignancies at Risk for Chemotherapy-Related Febrile Neutropenia. Integr. Cancer Ther. 2015, 14, 428-435. [CrossRef]

64. Tanaka, S.; Haruma, K.; Yoshihara, M.; Kajiyama, G.; Kira, K.; Amagase, H.; Chayama, K. Aged garlic extract has potential suppressive effect on colorectal adenomas in humans. J. Nutr. 2006, 136 (Suppl. S3), 821S-826S. [CrossRef]

65. Ishikawa, H.; Saeki, T.; Otani, T.; Suzuki, T.; Shimozuma, K.; Nishino, H.; Fukuda, S.; Morimoto, K. Aged garlic extract prevents a decline of NK cell number and activity in patients with advanced cancer. J. Nutr. 2006, 136 (Suppl. S3), 816S-820S. [CrossRef]

66. Wen-Qing, L.; Jing-Yu, Z.; Jun-Ling, M.; Li, Z.X.; Zhang, L.; Zhang, Y.; Guo, Y.; Zhou, T.; Ji-You, L.; Shen, L.; et al. Effects of Helicobacter pylori treatment and vitamin and garlic supplementation on gastric cancer incidence and mortality: Follow-up of a randomized intervention trial. BMJ 2019, 366, 15016.

67. Konmun, J.; Danwilai, K.; Ngamphaiboon, N.; Sripanidkulchai, B.; Sookprasert, A.; Subongkot, S. A phase II randomized double-blind placebo-controlled study of 6-gingerol as an anti-emetic in solid tumor patients receiving moderately to highly emetogenic chemotherapy. Med. Oncol. 2017, 34, 69. [CrossRef] [PubMed]

68. Hickok, J.T.; Roscoe, J.A.; Morrow, G.R.; Ryan, J.L. A phase II/III randomized, placebo-controlled, double-blind clinical trial of ginger (Zingiber officinale) for nausea caused by chemotherapy for cancer: A currently accruing URCC CCOP Cancer Control Study. Support. Cancer Ther. 2007, 4, 247-250. [CrossRef] [PubMed]

69. Nakayama, M.; Okizaki, A.; Takahashi, K. A Randomized Controlled Trial for the Effectiveness of Aromatherapy in Decreasing Salivary Gland Damage following Radioactive Iodine Therapy for Differentiated Thyroid Cancer. BioMed Res. Int. 2016, 2016, 9509810. [CrossRef]

70. Bossi, P.; Cortinovis, D.; Fatigoni, S.; Rocca, M.C.; Fabi, A.; Seminara, P.; Ripamonti, C.; Alfieri, S.; Granata, R.; Bergamini, C.; et al. A randomized, double-blind, placebo-controlled, multicenter study of a ginger extract in the management of chemotherapy-induced nausea and vomiting (CINV) in patients receiving high-dose cisplatin. Ann. Oncol. 2017, 28, 2547-2551.

71. Manusirivithaya, S.; Sripramote, M.; Tangjitgamol, S.; Sheanakul, C.; Leelahakorn, S.; Thavaramara, T.; Tangcharoenpanich, K. Antiemetic effect of ginger in gynecologic oncology patients receiving cisplatin. Int. J. Gynecol. Cancer 2004, 14, 1063-1069. [CrossRef]

72. Pillai, A.K.; Sharma, K.K.; Gupta, Y.K.; Bakhshi, S. Anti-emetic effect of ginger powder versus placebo as an add-on therapy in children and young adults receiving high emetogenic chemotherapy. Pediatric Blood Cancer 2011, 56, 234-238. [CrossRef]

73. Marx, W.; McCarthy, A.L.; Ried, K.; Vitetta, L.; McKavanagh, D.; Thomson, D.; Sali, A.; Isenring, L. Can ginger ameliorate chemotherapy-induced nausea? Protocol of a randomized double blind, placebo-controlled trial. BMC Complement. Altern. Med. 2014, 14, 134. [CrossRef]

74. Ansari, M.; Porouhan, P.; Mohammadianpanah, M.; Omidvari, S.; Mosalaei, A.; Ahmadloo, N.; Nasrollahi, H.; Hamedi, S.H. Eficacy of Ginger in Control of Chemotherapy Induced Nausea and Vomiting in Breast Cancer Patients Receiving Doxorubicin- Based Chemotherapy. Asian Pac. J. Cancer Prev. 2016, 17, 3877-3880.

75. Sanaati, F.; Najafi, S.; Kashaninia, Z.; Sadeghi, M. Effect of Ginger and Chamomile on Nausea and Vomiting Caused by Chemotherapy in Iranian Women with Breast Cancer. Asian Pac. J. Cancer Prev. 2016, 17, 4125-4129.

76. Panahi, Y.; Saadat, A.; Sahebkar, A.; Hashemian, F.; Taghikhani, M.; Abolhasani, E. Effect of Ginger on Acute and Delayed Chemotherapy-Induced Nausea and Vomiting: A Pilot, Randomized, Open-Label Clinical Trial. Integr. Cancer Ther. 2012, 11, 204-211. [CrossRef] [PubMed]

77. Lua, P.L.; Salihah, N.; Mazlan, N. Effects of inhaled ginger aromatherapy on chemotherapy-induced nausea and vomiting and health-related quality of life in women with breast cancer. Complement Ther. Med. 2015, 23, 396-404. [CrossRef] [PubMed] 
78. Thamlikitkul, L.; Srimuninnimit, V.; Akewanlop, C.; Ithimakin, S.; Techawathanawanna, S.; Korphaisarn, K.; Chantharasamee, J.; Danchaivijitr, P.; Soparattanapaisarn, N. Efficacy of ginger for prophylaxis of chemotherapy-induced nausea and vomiting in breast cancer patients receiving adriamycin-cyclophosphamide regimen: A randomized, double-blind, placebo-controlled, crossover study. Support. Care Cancer 2017, 25, 459-464. [CrossRef] [PubMed]

79. Li, X.; Qin, Y.; Liu, W.; Zhou, X.; Li, Y.; Wang, L. Efficacy of Ginger in Ameliorating Acute and Delayed Chemotherapy-Induced Nausea and Vomiting among Patients with Lung Cancer Receiving Cisplatin-Based Regimens: A Randomized Controlled Trial. Integr. Cancer Ther. 2018, 17, 747-754. [CrossRef] [PubMed]

80. Ryan, J.L.; Heckler, C.E.; Roscoe, J.A.; Dakhil, S.R.; Kirshner, J.; Flynn, P.J.; Hickok, J.T.; Morrow, G.R. Ginger (Zingiber officinale) reduces acute chemotherapy-induced nausea: A URCC CCOP study of 576 patients. Support. Care Cancer 2012, 20, 1479-1489. [CrossRef] [PubMed]

81. Arslan, M.; Ozdemir, L. Oral Intake of Ginger for Chemotherapy-Induced Nausea and Vomiting among Women with Breast Cancer. Clin. J. Oncol. Nurs. 2015, 19, E92-E97. [CrossRef]

82. Zick, S.M.; Ruffin, M.T.; Lee, J.; Normolle, D.P.; Siden, R.; Alrawi, S.; Brenner, D.E. Phase II trial of encapsulated ginger as a treatment for chemotherapy-induced nausea and vomiting. Support. Care Cancer 2009, 17, 563-572. [CrossRef]

83. Levine, M.E.; Gillis, M.G.; Koch, S.Y.; Voss, A.C.; Stern, R.M.; Koch, K.L. Protein and ginger for the treatment of chemotherapy-induced delayed nausea. J. Altern. Complement. Med. 2008, 14, 545-551. [CrossRef]

84. Marx, W.; McCarthy, A.L.; Ried, K.; McKavanagh, D.; Vitetta, L.; Sali, A.; Lohning, A.; Isenring, E. The Effect of a Standardized Ginger Extract on Chemotherapy-Induced Nausea-Related Quality of Life in Patients Undergoing Moderately or Highly Emetogenic Chemotherapy: A Double Blind, Randomized, Placebo Controlled Trial. Nutrients 2017, 9, 867. [CrossRef]

85. Evans, A.; Malvar, J.; Garretson, C.; Pedroja Kolovos, E.; Baron Nelson, M. The Use of Aromatherapy to Reduce Chemotherapy-Induced Nausea in Children With Cancer: A Randomized, Double-Blind, Placebo-Controlled Trial. J. Pediatric Oncol. Nurs. 2018, 35, 392-398. [CrossRef]

86. Liu, Y.Q.; Sun, S.; Dong, H.J.; Zhai, D.X.; Zhang, D.Y.; Shen, W.; Bai, L.L.; Yu, J.; Zhou, L.H.; Yu, C.Q. Wrist-ankle acupuncture and ginger moxibustion for preventing gastrointestinal reactions to chemotherapy: A randomized controlled trial. Chin. J. Integr. Med. 2015, 21, 697-702. [CrossRef] [PubMed]

87. Jafarpour-Sadegh, F.; Montazeri, V.; Adili, A.; Esfehani, A.; Rashidi, M.-R.; Mesgari, M.; Pirouzpanah, S. Effects of Fresh Yellow Onion Consumption on CEA, CA125 and Hepatic Enzymes in Breast Cancer Patients: A Double- Blind Randomized Controlled Clinical Trial. Asian Pac. J. Cancer Prev. 2015, 16, 7517-7522. [CrossRef] [PubMed]

88. Thomas, R.; Williams, M.; Sharma, H.; Chaudry, A.; Bellamy, P. A double-blind, placebo-controlled randomised trial evaluating the effect of a polyphenol-rich whole food supplement on PSA progression in men with prostate cancer-The UK NCRN Pomi-T study. Prostate Cancer Prostatic Dis. 2014, 17, 180-186. [CrossRef] [PubMed]

89. Paller, C.J.; Ye, X.; Wozniak, P.J.; Gillespie, B.K.; Sieber, P.R.; Greengold, R.H.; Stockton, B.R.; Hertzman, B.L.; Efros, M.D.; Roper, R.P.; et al. A randomized phase II study of pomegranate extract for men with rising PSA following initial therapy for localized prostate cancer. Prostate Cancer Prostatic Dis. 2013, 16, 50-55. [CrossRef]

90. Pantuck, A.J.; Pettaway, C.A.; Dreicer, R.; Corman, J.; Katz, A.; Ho, A.; Aronson, W.; Clark, W.; Simmons, G.; Heber, D. A randomized, double-blind, placebo-controlled study of the effects of pomegranate extract on rising PSA levels in men following primary therapy for prostate cancer. Prostate Cancer Prostatic Dis. 2015, 18, 242-248. [CrossRef]

91. Freedland, S.J.; Carducci, M.; Kroeger, N.; Partin, A.; Rao, J.Y.; Jin, Y.; Kerkoutian, S.; Wu, H.; Li, Y.; Creel, P.; et al. A Double-Blind, Randomized, Neoadjuvant Study of the Tissue Effects of POMx Pills in Men with Prostate Cancer before Radical Prostatectomy. Cancer Prev. Res. 2013, 6, 1120. [CrossRef]

92. Nuñez-Sánchez, M.A.; González-Sarrías, A.; García-Villalba, R.; Monedero-Saiz, T.; García-Talavera, N.V.; Gómez-Sánchez, M.B.; Sánchez-Álvarez, C.; García-Albert, A.M.; Rodríguez-Gil, F.J.; Ruiz-Marín, M.; et al. Gene expression changes in colon tissues from colorectal cancer patients following the intake of an ellagitannin-containing pomegranate extract: A randomized clinical trial. J. Nutr. Biochem. 2017, 42, 126-133. [CrossRef] 
93. Nuñez-Sánchez, M.A.; Dávalos, A.; González-Sarrías, A.; Casas-Agustench, P.; Visioli, F.; Monedero-Saiz, T.; García-Talavera, N.V.; Gómez-Sánchez, M.B.; Sánchez-Álvarez, C.; García-Albert, A.M.; et al. MicroRNAs expression in normal and malignant colon tissues as biomarkers of colorectal cancer and in response to pomegranate extracts consumption: Critical issues to discern between modulatory effects and potential artefacts. Mol. Nutr. Food Res. 2015, 59, 1973-1986. [CrossRef]

94. González-Sarrías, A.; Giménez-Bastida, J.A.; García-Conesa, M.T.; Gómez-Sánchez, M.B.; García-Talavera, N.V.; Gil-Izquierdo, A.; Sánchez-Álvarez, C.; Fontana-Compiano, L.O.; Morga-Egea, J.P.; Pastor-Quirante, F.A.; et al. Occurrence of urolithins, gut microbiota ellagic acid metabolites and proliferation markers expression response in the human prostate gland upon consumption of walnuts and pomegranate juice. Mol. Nutr. Food Res. 2010, 54, 311-322. [CrossRef]

95. Pantuck, A.J.; Leppert, J.T.; Zomorodian, N.; Aronson, W.; Hong, J.; Barnard, R.J.; Seeram, N.; Liker, H.; Wang, H.; Elashoff, R.; et al. Phase II Study of Pomegranate Juice for Men with Rising Prostate-Specific Antigen following Surgery or Radiation for Prostate Cancer. Clin. Cancer Res. 2006, 12, 4018-4026. [CrossRef]

96. Tajima, Y.; Ishida, H.; Yamamoto, A.; Chika, N.; Onozawa, H.; Matsuzawa, T.; Kumamoto, K.; Ishibashi, K.; Mochiki, E. Comparison of the risk of surgical site infection and feasibility of surgery between sennoside versus polyethylene glycol as a mechanical bowel preparation of elective colon cancer surgery: A randomized controlled trial. Surg. Today 2016, 46, 735-740. [CrossRef] [PubMed]

97. Agra, Y.; Sacristán, A.; González, M.; Ferrari, M.; Portugués, A.; Calvo, M.J. Efficacy of senna versus lactulose in terminal cancer patients treated with opioids. J. Pain Symptom Manag. 1998, 15, 1-7. [CrossRef]

98. Ramesh, P.R.; Kumar, K.S.; Rajagopal, M.R.; Balachandran, P.; Warrier, P.K. Managing morphine-induced constipation: A controlled comparison of an Ayurvedic formulation and senna. J. Pain Symptom Manag. 1998, 16, 240-244. [CrossRef]

99. Valverde, A.; Hay, J.M.; Fingerhut, A.; Boudet, M.J.; Petroni, R.; Pouliquen, X.; Msika, S.; Flamant, Y. Senna vs polyethylene glycol for mechanical preparation the evening before elective colonic or rectal resection: A multicenter controlled trial, French Association for Surgical Research. Arch. Surg. 1999, 134, 514-519. [CrossRef]

100. Tastekin, D.; Tambas, M.; Kilic, K.; Erturk, K.; Arslan, D. The efficacy of Pistacia Terebinthus soap in the treatment of cetuximab-induced skin toxicity. Investig. New Drugs 2014, 32, 1295-1300. [CrossRef]

101. Yayla, E.M.; Izgu, N.; Ozdemir, L.; Erdem, S.A.; Kartal, M. Sage tea-thyme-peppermint hydrosol oral rinse reduces chemotherapy-induced oral mucositis: A randomized controlled pilot study. Complement. Ther. Med. 2016, 27, 58-64. [CrossRef]

102. Charalambous, M.; Raftopoulos, V.; Paikousis, L.; Katodritis, N.; Lambrinou, E.; Vomvas, D.; Georgiou, M.; Charalambous, A. The effect of the use of thyme honey in minimizing radiation-induced oral mucositis in head and neck cancer patients: A randomized controlled trial. Eur. J. Oncol. Nurs. 2018, 34, 89-97. [CrossRef]

103. Charalambous, A.; Lambrinou, E.; Katodritis, N.; Vomvas, D.; Raftopoulos, V.; Georgiou, M.; Paikousis, L.; Charalambous, M. The effectiveness of thyme honey for the management of treatment-induced xerostomia in head and neck cancer patients: A feasibility randomized control trial. Eur. J. Oncol. Nurs. 2017, 27, 1-8. [CrossRef] [PubMed]

104. Jakab, F.; Shoenfeld, Y.; Balogh, A.; Nichelatti, M.; Hoffmann, A.; Kahán, Z.; Lapis, K.; Mayer, A.; Sapy, P.; Szentpetery, F; et al. A medical nutriment has supportive value in the treatment of colorectal cancer. Br. J. Cancer 2003, 89, 465-469. [CrossRef]

105. Demidov, L.V.; Manziuk, L.V.; Kharkevitch, G.Y.; Pirogova, N.A.; Artamonova, E.V. Adjuvant Fermented Wheat Germ Extract (Avemar(TM)) Nutraceutical Improves Survival of High-Risk Skin Melanoma Patients: A Randomized, Pilot, Phase II Clinical Study with a 7-Year Follow-Up. Cancer Biother. Radiopharm. 2008, 23, 477-482. [CrossRef]

106. Alberts, D.S.; Einspahr, J.; Rees-McGee, S.; Ramanujam, P.; Buller, M.K.; Clark, L.; Ritenbaugh, C.; Atwood, J.; Pethigal, P.; Earnest, D.; et al. Effects of Dietary Wheat Bran Fiber on Rectal Epithelial Cell Proliferation in Patients with Research for Colorectal Cancers. JNCI J. Natl. Cancer Inst. 1990, 82, 1280-1285. [CrossRef] [PubMed]

107. Garami, M.; Schuler, D.; Babosa, M.; Borgulya, G.; Hauser, P.; Müller, J.; Paksy, A.; Szabó, E.; Hidvégi, M.; Fekete, G. Fermented wheat germ extract reduces chemotherapy-induced febrile neutropenia in pediatric cancer patients. J. Pediatric Hematol. Oncol. 2004, 26, 631-635. [CrossRef] [PubMed] 
108. Bar-Sela, G.; Tsalic, M.; Fried, G.; Goldberg, H. Wheat Grass Juice May Improve Hematological Toxicity Related to Chemotherapy in Breast Cancer Patients: A Pilot Study. Nutr. Cancer 2007, 58, 43-48. [CrossRef] [PubMed]

109. von Hagens, C.; Walter-Sack, I.; Goeckenjan, M.; Storch-Hagenlocher, B.; Sertel, S.; Elsässer, M.; Remppis, B.A.; Munzinger, J.; Edler, L.; Efferth, T.; et al. Long-term add-on therapy (compassionate use) with oral artesunate in patients with metastatic breast cancer after participating in a phase I study (ARTIC M33/2). Phytomedicine 2019, 54, 140-148. [CrossRef] [PubMed]

110. Almubayedh, H.; Ahmad, R. Ethnopharmacological uses, phytochemistry, biological activities of Debregeasia salicifolia: A review. J. Ethnopharmacol. 2019, 231, 179-186. [CrossRef]

111. European Medicines Agency (EMA). Guideline on clinical investigation of medicinal products in the treatment or prevention of Diabetes Mellitus. Eur. Med. Agency 2012, 44, 1-28.

112. Ahmad, R.; Ahmad, N.; Al-Anaki, W.S.; Ismail, F.A.; Al-Jishi, F. Solvent and temperature effect of accelerated solvent extraction (ASE) coupled with ultra-high-pressure liquid chromatography (UHPLC-PDA) for the determination of methyl xanthines in commercial tea and coffee. Food Chem. 2020, 311, 126021. [CrossRef] [PubMed]

113. Ahmad, R.; Ahmad, N.; Shehzad, A. Solvent and temperature effects of Accelerated solvent extraction (ASE) coupled with Ultra-high pressure liquid chromatography (UHPLC-DAD) technique for determination of Thymoquinone in commercial food samples of black seeds (Nigella sativa). Food Chem. 2019, 309, 125740. [CrossRef]

114. Ahmad, R.; Ahmad, N.; Shehzad, A. Solvent and temperature effects of accelerated solvent extraction (ASE) with Ultra-high pressure liquid chromatography (UHPLC-PDA) technique for determination of Piperine and its ICP-MS analysis. Ind. Crops Prod. 2019, 136, 37-49. [CrossRef]

115. Vian, M.; Breil, C.; Vernes, L.; Chaabani, E.; Chemat, F. Green solvents for sample preparation in analytical chemistry. Curr. Opin. Green Sustain. Chem. 2017, 5, 44. [CrossRef]

116. World Health Organization (WHO). Operational Guidance: Information Needed to Support. Clinical Trials; World Health Organization: Geneva, Switzerland, 2005.

117. Ahmad, R. Current clinical status of osteopathy: Study based on retrospective evidences of six years, a systemic review. Annu. Res. Rev. Biol. 2017, 20, 1-20. [CrossRef]

118. Ahmad, R. Current Clinical Status of Homeopathy: An Evidence Based Retrospective Six Years Review. Annu. Res. Rev. Biol. 2018, 22,1-15. [CrossRef]

119. Furman, B.L.; Candasamy, M.; Bhattamisra, S.K.; Veettil, S.K. Reduction of blood glucose by plant extracts and their use in the treatment of diabetes mellitus; discrepancies in effectiveness between animal and human studies. J. Ethnopharmacol. 2020, 247, 2020. [CrossRef] [PubMed]

120. Zheng, W.; Chang, B.; Chen, J. Improving participant adherence in clinical research of traditional Chinese medicine. Evid.-Based Complement. Altern. Med. 2014, 2014. [CrossRef]

121. Ahmad, R.; Ahmad, N.; Amir, M.; Aljhisi, F.; Alamer, M.H.; Al-shaban, H.R.; Alsultan, B.M.; Alsadah, Z.A.; Aldawood, N.A.; Chathoth, S.; et al. Variation in Nigella sativa quality and its standardization via instrumental analysis: A study based on geographical origin. Not. Botanicae Horti Agrobot. Cluj-Napoca. 2020, 48, 1141-1154. [CrossRef]

122. Ahmad, R.; Ahmad, N.; Amir, M.; Aljishi, F.; Alamer, M.H.; Al-Shaban, H.R.; Alsadah, Z.A.; Alsultan, B.M.; Aldawood, N.A.; Chathoth, S.; et al. Quality Variation and standardization of black pepper (Piper nigrum): A comparative geographical evaluation based on instrumental and metabolomics analysis. Biomed. Chromatogr. 2019. [CrossRef]

123. Lacombe, D.; Liu, Y. The future of clinical research in oncology: Where are we heading to? Chin. Clin. Oncol. 2013, 2, 9.

124. Siu, L.L.; Ivy, S.P.; Dixon, E.L.; Gravell, A.E.; Reeves, S.A.; Rosner, G.L. Challenges and opportunities in adapting clinical trial design for immunotherapies. Clin. Cancer Res. 2017, 23, 4950-4958. [CrossRef]

125. Alonso-Castro, A.J.; del Carmen Juárez-Vázquez, M.; Campos-Xolalpa, N. Medicinal plants from Mexico, Central America, and the Caribbean used as immunostimulants. Evid. Based Complement. Altern. Med. 2016, 2016. [CrossRef] 
126. Riggio, C.; Pagni, E.; Raffa, V.; Cuschieri, A. Nano-oncology: Clinical application for cancer therapy and future perspectives. J. Nanomater. 2011, 2011. [CrossRef]

127. Shin, S.H.; Bode, A.M.; Dong, Z. Precision medicine: The foundation of future cancer therapeutics. NPJ Precis. Oncol. 2017, 1, 1-3. [CrossRef] [PubMed]

Publisher's Note: MDPI stays neutral with regard to jurisdictional claims in published maps and institutional affiliations.

(C) 2020 by the authors. Licensee MDPI, Basel, Switzerland. This article is an open access article distributed under the terms and conditions of the Creative Commons Attribution (CC BY) license (http://creativecommons.org/licenses/by/4.0/). 